\title{
La conduite de projets organisationnels portée par les cadres de santé : étude qualitative à partir d'un dispositif pédagogique.
}

\author{
Article pour la revue Recherche en Soins Infirmiers (2017)
}

The organizational projects management led by head nurses: qualitative study from an educational device.

Pierre-Philippe DUJARDIN - Infirmier, Ph.Dc, Cadre supérieur de santé, Institut de Formation des Cadres de Santé, CHU de Grenoble, laboratoire TIMC-IMAG / CNRS, Université Grenoble Alpes, France

Mail : ppdujardin@chu-grenoble.fr

Tél : 04.76.76.51.27

Thomas REVERDY - Sociologie, Ph.D, Maître de conférences, laboratoire PACTE / CNRS, Université Grenoble Alpes, France

Annick VALETTE - Science de gestion, Ph.D, Maître de conférences, laboratoire CERAG / CNRS, Université Grenoble Alpes, France

Patrice FRANÇOIS - Médecin, Ph.D, Professeur, Unité d'Evaluation Médicale, CHU de Grenoble, laboratoire TIMC-IMAG / CNRS, Université Grenoble Alpes, France

\section{Résumé}

Le travail de coordination opérationnelle par les Cadres de Santé (CdS) est mieux connu que leurs capacités de transformation de l'organisation du travail et des pratiques de soin. Dans cet article, nous étudions des projets organisationnels conduits par des CdS qui peuvent, selon les cas, concerner leur équipe, impliquer la hiérarchie hospitalière ou d'autres équipes. Nous nous appuyons sur une recherche-intervention qui a permis de suivre 17 projets organisationnels portés par des CdS sur une année. Les résultats de cette recherche mettent en valeur les efforts d'intercompréhension de la part des CdS vis-à-vis des autres acteurs comme la principale condition de réussite. Les $\mathrm{CdS}$ qui sollicitent la participation de leur équipe à l'élaboration des solutions obtiennent des améliorations durables. Quand les problèmes identifiés impliquent la hiérarchie, les $\mathrm{CdS}$ cherchent à développer des relations interpersonnelles. Quand ils impliquent d'autres services dont ils sont dépendants, les CdS engagent des négociations intégratives. Ces résultats suggèrent de développer la discussion sur l'organisation du travail et de contractualiser les projets organisationnels. Cette rechercheintervention permet aussi de valider la pertinence d'une formation-action des $\mathrm{CdS}$ au diagnostic organisationnel.

Mots-clefs : Cadre de santé ; Qualité ; Management ; Organisation du travail ; Gestion de projet

\section{Abstract}

The work of operational coordination led by head nurses (HN) is more known than their abilities to change work organization, as well as, care practices. In this article, we explore organizational projects led by HN. Depending on the situations, they may concern their health care team or involve institutional hierarchy or other teams. We rely on an intervention study, which enabled us to follow 17 organizational projects led by $\mathrm{HN}$ for one year. The results of this study highlight as the main condition for success, the $\mathrm{HN}^{\prime}$ efforts to enable mutual understanding with other interested actors. Managers, who engage their health care team in the development of solutions, bring sustainable results. When problems involve the hierarchy, 
the managers focus on the development of personal relationships. When problems involve other departments, which they depend on, then they engage themselves into integrative negotiations. These results invite to open discussion about work organization and to formalize organizational projects. This intervention study also points out that work - training at operational audit is relevant for $\mathrm{HN}$.

Keywords : Head nurse ; Quality ; Management ; Work organization ; Project management

\section{Introduction}

Le manager en général et le cadre de santé $(\mathrm{CdS})$ en particulier est considéré comme un pivot organisationnel (1-3). On compte sur lui pour réguler les ambiguités, les contradictions $(4,5)$ et articuler les logiques d'action parfois antinomiques (3,6-11). Au-delà d'articuler les éléments, son activité se développe dans deux temporalités différentes. Le manager opère une coordination opérationnelle au quotidien pour résoudre les problèmes urgents, ajuster les ressources aux besoins pour tenter de préserver la fluidité de l'activité. Il développe également une coordination organisationnelle à moyen ou long terme pour mettre en œuvre des projets et conduire le changement.

Le rôle central du CdS dans la coordination opérationnelle a fait l'objet de descriptions précises $(4,12)$, sans pour autant s'intéresser aux relations de pouvoir et d'autorité. En conséquent, on sait peu de choses sur sa capacité d'action comme acteur du changement. Mucchielli indique qu'il ne s'occupe pas de l'organisation du travail (13). Detchessahar et Grévin montrent qu'il est pris dans une spirale gestionnaire institutionnelle qui l'éloigne de sa fonction de proximité $(14,15)$. François et al. l'identifient comme un entrepreneur local du changement mais qui manque de soutien (16).

Nous sommes ainsi face à une forme de paradoxe. L'activité de coordination organisationnelle est valorisée par les décideurs $(17,18)$, les chercheurs $(3,8,11,19-21)$, et les CdS eux-mêmes (22). Pour autant, cette activité de coordination reste mal connue dès lors qu'elle sort de l'activité opérationnelle quotidienne. L'évaluation de ses capacités de coordination est problématique car celle-ci s'inscrit dans trois directions relationnelles (5) où le CdS ne possède pas le même pouvoir : en proximité dans l'unité dont le manager a la charge, en transversal avec d'autres partenaires pour la prise en charge clinique des patients, vers le reste de l'organisation hiérarchique, administrative ou médicale dont dépend le CdS.

Notre projet de recherche propose d'approfondir ce constat et d'explorer la capacité d'action organisationnelle des cadres en s'appuyant sur un dispositif pédagogique de formation des cadres de santé. Des CdS confient à des étudiants, un problème d'organisation concret. Après une période de travail dans l'unité, les étudiants remettent au CdS un rapport écrit qui reprend l'analyse et des propositions d'actions d'amélioration. L'ensemble de cette démarche confiée à un tiers est intitulé Projet Qualité Tutoré (PQT).

Ces PQT représentent une opportunité pour identifier et comprendre le rôle de coordination organisationnelle du CdS. Les PQT se distinguent de l'activité quotidienne. Ils renvoient à des formes de participation et d'interaction dans trois dimensions relationnelles : à l'intérieur du service, avec certains interlocuteurs d'autres services, avec le reste de l'organisation. La gestion de projet est approchée comme un révélateur de l'activité des $\mathrm{CdS}$ dans la conduite du changement.

Cette recherche a déjà fait l'objet d'un premier travail quantitatif pour mesurer l'impact des actions mises en œuvre par le CdS, à partir de 12 variables d'influence (23). Les résultats 
montrent que les CdS ont identifié une amélioration effective dans $71 \%$ des actions mises en œuvre. Cette approche originale a permis de distinguer les facteurs d'influence de l'action des $\mathrm{CdS}$. Le succès dépendait de la nature de l'action, plus fréquent pour les actions d'organisation ou l'accompagnement des équipes par rapport aux actions d'équipement. Il était fonction de la manière dont le $\mathrm{CdS}$ gérait les aléas, moins fréquent quand le $\mathrm{CdS}$ laissait faire par rapport au fait de tenir la trajectoire de l'action. L'objectif aujourd'hui est de compléter cette approche quantitative par une analyse qualitative pour cerner les stratégies, les méthodes utilisées par les $\mathrm{CdS}$ et leur capacité à transformer l'organisation.

\section{Cadre théorique}

Pour percer les mécanismes de transformation organisationnelle, la revue bibliographique prend, dans un premier temps, appui sur la sociologie des organisations et l'existence d'une diversité de logiques en relation d'interdépendance. Ensuite, la sociologie de l'innovation permet de rendre compte des conditions pour conduire le changement organisationnel. Ce cadre d'analyse est enfin enrichi par une identification des compétences de leadership nécessaires au promoteur de changement, ainsi que la place des espaces de discussion pour faciliter l'appropriation.

\section{Faire converger des logiques diversifiées}

Selon la sociologie des organisations, la difficulté dans l'activité de coordination organisationnelle réside dans la capacité à concilier les acteurs interdépendants, aux pouvoirs, logiques et enjeux différents $(11,24)$. Ce postulat conduit à poser deux principes fondamentaux.

- D'une part, il est illusoire de penser que naturellement chacun va accepter de se soumettre à une autorité et à un principe organisateur commun qui viendraient réduire sa marge de manœuvre. Le manager lui-même cherche à définir son propre rôle dans l'organisation existante pour préserver son autonomie (2).

- D'autre part, chacun pour conduire son action est dépendant et en interaction avec d'autres acteurs dans un système structuré. Dans ce contexte la régulation managériale ne peut se limiter à la dimension hiérarchique, elle est aussi adaptative au contexte (2).

Ainsi, l'action collective est une coalition d'acteurs dont la situation rend l'intention convergente. En fonction de ses convictions et de son objectif, chacun exploite les opportunités et les contraintes du contexte pour développer son pouvoir d'action, son autonomie, tout en cherchant à réduire les dépendances.

La littérature montre que dans les organisations hospitalières, malgré des interdépendances fortes entre les acteurs et les différents services, chacun conserve une grande autonomie $(1,6,9,11-13)$. Plusieurs formes de pouvoir se diffusent, notamment du fait d'une hiérarchie tricéphale (administrative, médicale et soignante), d'une réglementation procédurale importante et de compétences professionnelles elles-mêmes réglementées. Cela provoque le développement d'une multitude de normes qui ne sont pas régulées collectivement (25). La régulation est laissée à la responsabilité des acteurs (10). Cette structuration induit des cloisonnements qui limitent le processus de changement organisationnel.

Mucchielli identifie qu'au-delà de l'organigramme qui pose les relations hiérarchiques, les charges de responsabilité restent floues (26). Il identifie «quatre pathologies principales essentiellement liées à l'encadrement : le management hyper-affectif, la défaillance des 
cadres soignants, le dévouement paradoxal, le dysfonctionnement des services. »(26) La fonction du $\mathrm{CdS}$ est représentée comme un sacerdoce basé sur une forte implication professionnelle, une gestion émotionnelle, une soumission au pouvoir médical et une domination par les subordonnés soignants. Comme a pu le montrer Buscatto (27) dans le secteur des assurances, le manager issu du terrain serait dans l'impossibilité de remplir ses fonctions. Le CdS manquerait de confiance et serait en incapacité d'évaluer, de décider et de gérer les conflits (26). Il ne ferait pas autorité (16) et serait au contraire l'otage d'enjeux institutionnels (13), préférant faire «le dos rond» face à cette complexité (26). Dans ce contexte, Burelier identifie au contraire que le CdS serait en capacité de «tirer son épingle du jeu » en développant des stratégies en marge des injonctions pour développer son pouvoir d'action (28).

Ainsi, en s'appuyant sur la sociologie des organisations, la conduite du changement doit faire face à une double pression entre réduire les incertitudes pour sécuriser les pratiques et prendre en compte l'autonomie des acteurs. En conséquence, on peut imaginer que le succès de la conduite de changement dépend avant tout de la considération portée aux intérêts individuels et collectifs à modifier la pratique.

\section{Le rôle du traducteur pour articuler les logiques différentes}

Toute transformation organisationnelle débute par la volonté d'un «entrepreneurship », comme le nomme Friedberg (24), à modifier l'équilibre de l'environnement existant $(2,9)$. Celui-ci se fixe un objectif, identifie une trajectoire et un plan d'action pour atteindre son but (29). Cet entrepreneur peut ensuite conduire son projet de deux manières résolument différentes.

- Soit il reste centré sur son projet et sa logique à partir d'arguments techniques qu'il aura lui-même identifiés. C'est ce que Callon nomme le modèle de la diffusion. L'implantation du changement dépend alors du pouvoir de l'entrepreneur à imposer sa rationalité aux plus faibles.

- Soit il prend en compte les attentes de chacun et ajuste son projet. C'est le modèle de l'intéressement. L'objectif n'est pas de résoudre le problème mais de chercher à le comprendre.

Or, la coordination collective est une activité de décloisonnement dont le succès dépend avant tout «de l'agrégation d'intérêts » (30) dont va dépendre l'intensité des relations entre les acteurs et groupes d'acteurs (31). La conduite du changement ne peut se faire sans coalition d'acteurs (9). La sociologie de l'innovation identifie trois éléments principaux.

L'intéressement consiste à identifier l'intérêt d'une personne ou d'un groupe de personnes. C'est se mettre à la place des autres, exploiter les bénéfices escomptés pour ces personnes, tout en cherchant à limiter les contraintes. Car c'est la perspective d'un gain qui permet d'envisager un déplacement $(2,32)$. Le traducteur est celui qui joue sur les deux tableaux : «celui qui met en relation deux univers aux logiques et aux horizons distincts, deux mondes séparés mais qui ne sauraient vivre l'un sans l'autre. »(30). L'objectif du traducteur est de rendre le contexte favorable à l'action collective (33), construire le réseau d'alliance nécessaire et mettre à l'écart les éléments qui viendraient perturber le projet collectif (32). Pour jouer ce rôle la connaissance de l'environnement est un atout indispensable.

Mais l'intéressement n'est pas suffisant pour provoquer le mouvement. L'enrôlement est plus fort car il traduit l'implication des protagonistes dans le changement (32). Engager l'autre c'est lui accorder une place, lui attribuer un rôle, une participation active qui se traduira ensuite en action. 
La confiance réciproque constitue le troisième déterminant. La diversité des enjeux, des objectifs visés, des rationalités préalables définissent un contexte général instable et en perpétuel mouvement. La défaillance d'un protagoniste sur l'objectif à atteindre, ou les moyens pour atteindre cet objectif, seront pris comme des actes de trahison qui peuvent réduire à néant tous les investissements antérieurs $(11,32)$. Chacun repartira dans une logique propre à ses intérêts (24).

Traduire c'est redonner du sens (25), être capable de recombiner un travail divisé pour en faire une activité collective (30), construire des interactions et du décloisonnement pour développer de nouveaux couplages favorables au changement visé (33). C'est un processus long, itératif et fragile dont le résultat n'est jamais assuré (11,31). D'ailleurs à propos du management, Mintzberg dit que l'échec est toujours garanti alors que le succès ne s'obtient qu'après de longs efforts (5), traduisant par-là la difficulté à pouvoir construire ces couplages et les tenir dans le temps.

\section{La place du leadership dans la conduite du changement}

L'approche développée dans la sociologie de l'innovation est une représentation des échanges qui n'intègre pas la dimension psychologique. Elle met de côté la reconnaissance et les dynamiques de leadership. D'autres auteurs apportent des compléments en explorant les interrelations entre l'acteur qui induit le changement et les autres en charge de l'appliquer.

En analysant les conditions d'implantation d'une nouvelle technique chirurgicale coronarienne micro-invasive, Edmonson et al. (34) montrent l'impact du processus d'intercompréhension sur le résultat de l'implantation de la technique. L'implication des équipes chirurgicales dans le processus de sélection des professionnels, dans la formation et avec des relations symétriques lors des briefings et débriefings de chaque intervention, conditionnent l'implantation de la nouvelle technique. A l'inverse celles qui sont restées centrées sur l'évolution technologique, sans apporter de considération spécifique aux autres acteurs concernés par ce changement, n'ont pas réussi à implanter la technique.

On retrouve des résultats similaires dans l'étude d'Habib et al. (9) concernant le rôle du directeur d'hôpital dans la conduite de projet d'établissement. L'étude de cas montre que la cohésion prime sur l'injonction de changement. Le succès est lié au maintien du lien social. Il dépend de l'ancrage aux préoccupations des acteurs de terrain, à la gestion des temporalités, à l'animation d'espaces de discussion et de décision. A l'inverse se réfugier derrière la pression des tutelles et mettre à distance les professionnels, provoquent d'une part des injonctions irréalistes et contradictoires, d'autre part des résistances et un désengagement des professionnels qui se sentent déconsidérés. Ainsi cet article réaffirme que l'objectif managérial doit autant s'attacher à produire de la valeur symbolique en redonnant du sens à l'action collective, qu'à produire de la valeur économique dans une quête de performance.

Ces deux articles montrent que la seule autorité du leader, qu'il soit médecin ou directeur, n'est pas suffisante. Ces expériences traduisent explicitement que la croyance d'une expertise détenue par un seul et même acteur constitue la garantie d'un échec. Le pouvoir de prescription d'un chef, pour transformer l'organisation, n'est pas suffisant. Négocier, c'est accepter de débattre, écouter l'autre et considérer ses arguments pour construire ensemble une convention collective. En pratique cela demande du temps d'accepter de laisser de côté sa propre rationalité professionnelle, car généralement, les solutions sont pensées avant même la formulation du problème (33). Chacun a des idées pour résoudre le problème, sans penser que ces formulations sont antagoniques avec les autres logiques professionnelles.

Cette position de traducteur nécessite de jouer sur plusieurs tableaux dans une démarche d'intercompréhension. Or, le CdS est aussi un ancien expert en soin, issu du milieu 
professionnel et toujours attaché aux valeurs soignantes $(1,3,22,28)$. Cette antériorité lui donne l'atout d'une bonne connaissance de l'environnement dans lequel il évolue. Mais cette expertise technique influence aussi la représentation du rôle d'encadrement (27) et sa capacité à se décaler d'un savoir technique avec une vision professionnelle et une démarche prescriptive, vers celui de chef de projet avec une vision globale et une démarche fédérative (35).

\section{Les espaces de discussion dans la conduite du changement}

Edmonson et Habib démontrent que le succès dépend d'une démarche de coconstruction interprofessionnelle. Le leader développe des relations symétriques avec l'environnement, partage les connaissances, considère les contraintes respectives, négocie les perspectives et partage les décisions. Comme le rappelle Minzberg, la coordination collective ne s'impose pas (5). La régulation managériale n'est pas que hiérarchique, elle est aussi adaptative au contexte $(2,11)$. Pour ce faire, elle doit passer par des espaces de discussion (36) dont la fonction est de penser, de partager et de négocier l'organisation (35).

Or Detchessahar et Grévin montrent que les CdS sont pris dans une spirale gestionnaire entre activité de reporting et réunions. Cette aspiration vers le haut a pour effet de délaisser le management de proximité sans pour autant récupérer un pouvoir d'influence au niveau institutionnel $(14,15,35)$. Lépine ajoute que les $\mathrm{CdS}$ développent peu d'espaces de discussion formalisés (37). Les discussions sont de nature informelle et interpersonnelle, destinées à trouver des micro-ajustements pour une coordination opérationnelle $(4,12)$.

\section{Objectif de l'étude}

Ainsi, pour apprécier la posture du CdS dans l'activité de coordination organisationnelle, l'étude s'est portée sur le suivi de la mise en œuvre des PQT, après l'analyse et la proposition d'un plan d'action produit par un groupe d'étudiants cadres. Il s'agissait d'identifier la capacité d'action du CdS à transformer l'organisation.

\section{Méthode}

\section{Intervention à partir d'un dispositif pédagogique}

L'étude s'est adossée sur un dispositif pédagogique construit pour des étudiants cadres, en partenariat avec les CdS du Centre Hospitalier Universitaire de Grenoble Alpes (CHUGA). L'établissement a une population d'environ $150 \mathrm{CdS}$, répartis dans une quinzaine de pôles cliniques, pour une capacité de 2200 lits.

Tous les ans depuis 2009, l'Institut de Formation des Cadres de Santé (IFCS) de Grenoble propose aux CdS de soumettre un problème organisationnel. Chaque proposition, une dizaine par an, est travaillée par un groupe de quatre ou cinq étudiants.

Le dispositif pédagogique se compose de quatre journées dans le service, entre décembre et mars, pour étudier le problème. Les étudiants sont accompagnés par trois enseignants : un sociologue en génie industriel, un ingénieur en organisation et méthode et un formateur responsable du dispositif pédagogique et investigateur de la recherche. L'analyse des données conduit à la formulation d'un plan d'action destiné à corriger le problème. L'intervention des étudiants se termine par la restitution au CdS d'un rapport écrit. L'ensemble de la démarche est nommé PQT (38). 


\section{Population}

Pendant les trois années universitaires de 2011 à 2014, 28 PQT ont été menés. Chaque PQT a fait l'objet d'un suivi sur une période d'un an, pour identifier la capacité d'action du $\mathrm{CdS}$ à transformer l'organisation. Tous les $\mathrm{CdS}$ ont accepté de participer à la recherche, mais 11 PQT sont sortis de l'étude suite au départ du CdS de l'unité dans l'année de suivi. Au final l'étude a porté sur 17 PQT.

\section{Recueil de données}

Le suivi des PQT s'est fait par des entretiens semi-directifs conduits de façon indépendante auprès des $\mathrm{CdS}$ et des représentants de l'équipe. Pour ces derniers, il s'agissait de soignants volontaires et disponibles au moment de l'entretien dont le rendez-vous était fixé par le CdS. Cette triangulation avait pour objectif d'améliorer la validité des discours.

Ces entretiens ont été conduits à deux moments différents. Le premier, deux mois après la remise du PQT au CdS $(\mathrm{M}+2)$, a identifié les bénéfices du travail et les perspectives de mise en œuvre. Le second, un an plus tard $(\mathrm{M}+12)$, a exploré les conditions de transformation de l'organisation et la formulation du résultat.

Les entretiens ont été enregistrés après obtention de l'autorisation des participants. La retranscription fut complète pour les entretiens auprès des $\mathrm{CdS}$ et partielle pour ceux des représentants de l'équipe.

\section{Analyse}

Les PQT ont été définis à partir des préoccupations des CdS. Ils ont constitué une collection d'objets hétérogènes : fonctions logistiques, organisation des soins, gestion des ressources humaines, qualité et gestion des risques. Ils ont également impliqué une diversité d'acteurs : soignants, médicaux, administratifs et supérieurs hiérarchiques du $\mathrm{CdS}$, avec pour chacun une relation spécifique pour traiter la question de la coordination organisationnelle. Ainsi, chaque PQT a suivi une trajectoire spécifique.

L'analyse de contenu s'est effectuée par le codage du matériau s'est réalisé à l'aide du logiciel N-vivo 9, selon une approche inductive (39). Les hypothèses de recherche et le cadre d'analyse ont été formulés à partir des données collectées dans la tradition de la théorie ancrée de Glasser \& Strauss (40). Il est proposé de développer les différentes étapes qui ont permis de passer du matériau brut à l'exploitation structurée des données (41).

Une fois les entretiens retranscrits, les documents ont été importés dans la base. Le titre des documents identifiait trois éléments: le $\mathrm{n}^{\circ}$ du PQT, le profil de l'enquêté (CdS ou soignant), le moment de l'entretien $(\mathrm{M}+2$ ou $\mathrm{M}+12)$. Ces paramètres d'identification sont retrouvés devant les verbatim au moment du requêtage de la dernière étape.

Avant de débuter le codage quelques catégories génériques sont identifiées pour explorer le matériau (Tableau 1) : 1) les comportements des CdS ; 2) les comportements des soignants et autres partenaires ; 3) les outils et méthodes de travail ; 4) les conditions de mise en œuvre et trajectoires des PQT. Ces catégories sont pensées à partir des grilles d'entretiens utilisées pour le recueil de données.

L'exploitation du matériau a consisté ensuite à sélectionner successivement des unités d'enregistrement (verbatim) et de les lier à un ou plusieurs codes. Une unité d'enregistrement correspond à une sélection du corpus de données dont la taille allait de quelques mots à plusieurs phrases en référence à une unité de sens. Deux alternatives sont possibles. Soit le code a préalablement été identifié et il suffit alors de glisser la sélection de texte vers le code 
pour établir le codage. Soit un nouvel élément, qui ne peut être classé dans un des codes existants, émerge. Un nouveau code est alors créé avant d'affecter la sélection. Un verbatim peut être référencé à plusieurs codes. Par exemple (Tableau 1), la mise en œuvre d'une action par le CdS (P06.01.01) renvoyait éventuellement à la question de la gestion des temporalités (M02.02), mais également à l'expression d'une conception managériale (C08), d'une approche organisationnelle (C09), ou communicationnelle (C10). Elle a pu traduire la capacité d'action du CdS (C04) mais pouvait également laisser entrevoir la place accordée aux autres partenaires (S04). Ce codage et les relations entre les codes à partir d'un corpus de données étaient spécifiques à chaque verbatim. Ce maillage est ensuite exploité pour l'analyse.

Les codes sont les éléments de base, qui représentent les différentes modalités rencontrées. L'opération de catégorisation consiste ensuite à regrouper sous un intitulé générique plusieurs codes de même genre. Par exemple le suivi de la mise en œuvre des PQT a laissé apparaitre différentes formes de management (C08) : participatif, délégatif ou directif en fonction des situations rencontrées et des acteurs concernés. La catégorisation s'opère progressivement pendant le travail de codage. Elle permet de ranger les codes et de distinguer progressivement une arborescence par regroupement des codes en sous-thèmes eux même en référence à des thèmes. Mais si un verbatim peut être affecté à plusieurs codes, le code ne peut être affecté qu'à une seule catégorie.

Le travail de codage est un processus long et inductif qui conduit à une diversité de codes qui peuvent parfois correspondre à des synonymes. Une fois le codage terminé pour l'ensemble du matériau, la cohérence de la base de données est à examiner. Cette étape consistait à vérifier l'arborescence et réaffecter certains verbatim à des codes pour supprimer les doublons.

Le codage est une immersion dans les données. Il donne une connaissance fine du matériau qui permet de formuler des hypothèses. La catégorisation donne ainsi une représentation simplifiée du corpus de données. Elle permet de faire des requêtes, par sélection d'un ou plusieurs codes et de comparer les unités d'enregistrement. L'analyse se fait par comparaison successive des éléments dans l'objectif d'identifier certains raisonnements et logiques d'action, jusqu'à la formulation des résultats. Le matériau a été analysé pour mettre en valeur la capacité de coordination organisationnelle du CdS. Le croisement des données a révélé des différences en fonction des dimensions relationnelles et du niveau d'intercompréhension entre les acteurs.

L'analyse a fait émerger les trois dimensions relationnelles identifiées dans le modèle proposé par Mintzberg (5) : a) une gestion de proximité, dans l'unité dont le CdS a la responsabilité en lien avec les relations internes, b) une gestion institutionnelle, vers le reste de l'organisation pour traduire les relations avec les pouvoirs, hiérarchique, administratif ou médical, c) une gestion transversale, avec les partenaires impliqués dans la prise en charge clinique des patients (Figure 1). En fonction des acteurs concernés, il s'agissait de distinguer les relations d'autorité et de pouvoir, les logiques d'action sous-tendues, l'autonomie du CdS ainsi que les formes de négociation que celui-ci était en capacité de développer dans le contexte.

L'analyse a permis, pour chaque PQT de produire une monographie simplifiée pour rendre compte des trajectoires du PQT. Elles partaient des demandes initiales des CdS jusqu'à la formulation d'un résultat. 


\section{Résultats}

L'enquête montre que les CdS formulent les problèmes et construisent les solutions en prenant en considération le contexte organisationnel dans lequel ils se placent. Ils font preuve de compétences stratégiques en identifiant les acteurs et les ressources techniques dont ils dépendent et en élaborant des stratégies pour les mobiliser. La trajectoire du PQT est révélatrice de ces compétences stratégiques et de ces capacités d'action. Néanmoins, dans le détail des trajectoires étudiées, on observe des variations dans la façon dont les CdS tentent d'impliquer les autres acteurs. Les stratégies qu'ils emploient sont diversifiées et ont plus ou moins de succès.

La présentation des résultats distingue les trois principales directions dans lesquelles les $\mathrm{CdS}$ investissent pour nouer les relations nécessaires à leur projet de changement: la mobilisation des professionnels de leur équipe, l'implication des responsables hiérarchiques et fonctionnels, la négociation avec les autres services de soins. Pour chacune de ces directions les stratégies d'intéressement et d'enrôlement ne sont pas les mêmes. Les capacités d'action du CdS, ses enjeux managériaux et son leadership sont différents.

\section{La mobilisation des équipes dans la conduite du changement}

La première direction relationnelle étudiée concerne le fonctionnement interne à l'unité et l'équipe dont le $\mathrm{CdS}$ a la responsabilité hiérarchique. L'analyse montre une diversité dans la manière de mobiliser les équipes pour atteindre l'objectif de transformation du PQT. Elle se regroupe autour de deux modèles opposés, une posture inter-compréhensive versus une posture prescriptive ${ }^{1}$, que nous pouvons compléter par une posture intermédiaire qualifiée d'inter-compréhensive asymétrique.

\section{Une posture inter-compréhensive}

Un premier groupe de CdS a adopté une posture que l'on peut qualifier d'intercompréhensive. Nous proposons ici de caractériser en détail cette posture à partir de l'analyse des pratiques managériales, telle qu'elles sont décrites par les CdS et relatées par les membres des équipes interrogées.

Tout d'abord, le CdS cherche l'accord avec l'équipe sur la manière de conduire le PQT avant même de chercher à résoudre le problème, dans une volonté d'intercompréhension. Pour ce faire les PQT se travaillent habituellement en réunion d'équipe. Des groupes de travail sont organisés pour la rédaction de procédures.

Dans un premier temps les CdS animent des débats avec l'équipe autour des propositions d'actions contenues dans le PQT. Ils ne font pas valoir une position autoritaire, mais au contraire une relation de symétrie avec l'équipe à partir des arguments de chacun. Les $\mathrm{CdS}$ utilisent leurs connaissances professionnelles pour échanger avec l'équipe sans pour autant imposer leur rationalité. Cette posture managériale inter-compréhensive conduit stratégiquement certains $\mathrm{CdS}$ au renoncement temporaire de certaines actions invalidées par l'équipe comme l'illustre le PQT 04 (Encadré 1). Pour autant ces CdS ne considèrent pas que leur autorité se trouve remise en question. Ils exprimaient très clairement qu'aller dans le sens de l'équipe ne signifie pas se soumettre à elle, mais avancer ensemble : "Je voulais que l'idée vienne d'elles [les soignantes] sur ce qui est à mettre en place [...]. Parce qu'elles sont

\footnotetext{
${ }^{1}$ La posture prescriptive est à prendre dans le sens organisationnel. Il ne s'agit en aucun cas d'une prescription de nature médicale.
} 
d'accord, mais dans les faits, il y a un écart entre le "je suis d'accord » et "je le mets en œuvre ». » La volonté managériale est d'enrôler l'équipe dans la démarche de changement.

Par rapport à la posture prescriptive qui sera présentée ensuite, deux facteurs ont caractérisés ces CdS, d'une part la place accordée aux espaces de discussion collectifs, d'autre part la considération apportée à la dimension temporelle. Ils montrent une capacité de résistance à la pression générale de l'urgence et de l'action immédiate. Les CdS se donnent du temps pour que les réflexions, issues des débats, puissent maturer individuellement et collectivement. Ils orchestrent une alternance entre des temps formels de réunions et des temps informels d'échanges interindividuels pour négocier et travailler des résistances révélées en réunion : "Pour les équipes, il faut donner beaucoup d'explications, il faut discuter, il faut faire des réunions, il faut faire participer pour que les choses avancent. C'est un petit peu lent, [...] parce que pour soi-même ça pourrait se régler beaucoup plus vite. [...]. C'est du travail, c'est du long terme.»

Quelles que soient les possibilités offertes par l'organisation, les CdS accordent une place spécifique à la communication collective. Dans les contextes où l'organisation du travail limite la possibilité d'instaurer des réunions, plusieurs CdS font progresser leur PQT dans le cadre des animations à intervalles courts qu'ils ont mises en place dans le service. Tous les jours à la même heure, l'équipe et le CdS se réunissent dix minutes autour du tableau d'activité pour échanger sur les questions d'organisation. Les CdS ont mis en avant l'intérêt de cette brièveté qui rend ces «mini-réunions » compatibles avec l'activité de soins. La récurrence journalière permet de coordonner les activités collectives, de rappeler certains messages, de suivre la mise en œuvre des actions. Les PQT sont abordés dans ces espaces de discussion.

Les équipes rencontrées ont systématiquement validé l'existence et l'importance des problèmes définis initialement par les CdS. L'engagement collectif est retrouvé dans les propos des professionnels qui ont été en capacité de présenter le travail réalisé autour du PQT. Ils ont identifié les liens entre les actions et les bénéfices escomptés. Ils ont pu parler des alternatives discutées collectivement et des décisions prises. Au final les discours des professionnels ont montré une conception partagée de la conduite du changement dans laquelle ils s'étaient engagés.

Le leadership du CdS dans sa fonction d'animateur est identifié comme une caractéristique positive pour l'équipe. Les discussions collectives permettent de clarifier les organisations et d'apaiser les relations de travail de l'équipe.

\section{Encadré 1 : Réduire le retard des consultations pour les patients handicapés (PQT 04)}

L'unité d'appui est chargée de fournir des professionnels accompagnants pour les consultations de patients handicapés. Une des difficultés majeures est d'être informé par les services de consultations en cas de modification de programmation, alors que le dossier du patient handicapé est identique à tous les autres. Cette défaillance communicationnelle perturbe la coordination. Le CdS a proposé de mettre une pastille sur les dossiers. Ce signe distinctif permettrait d'alerter les consultations sur la spécificité du rendez-vous et la nécessité de prévenir l'unité d'appui en cas de modification. L'équipe refuse catégoriquement cette proposition qui stigmatise cette population. Le $\mathrm{CdS}$ reconnait l'argument et il ne donne pas suite à son action. Par contre, lorsque le dysfonctionnement se présente, il le relate à l'équipe, argumentant avec habileté qu'une pastille sur le dossier aurait évité cet incident dommageable pour le patient. Un an plus tard, l'équipe propose elle-même qu'une pastille soit collée sur le dossier des patients handicapés. 
L'analyse identifie une posture inter-compréhensive dans sept PQT (Tableau 2). Elle se retrouve chez les CdS issus des métiers de la rééducation (2/2), infirmier (4/11) et accessoirement médico-technique (1/4).

\section{Une posture prescriptive}

L'analyse identifie un second groupe de CdS, qui adopte une posture que l'on pourrait qualifier de prescriptive. Pour ces CdS l'important est de résoudre le problème dans les plus brefs délais. Ils élaborent seuls le projet et sa mise en œuvre, à partir de leurs compétences organisationnelles et soignantes. Ils ne veulent pas imposer les choses, mais cherchent plutôt à protéger l'équipe déjà surchargée, d'un travail supplémentaire. Pour ces CdS, leur conception organisationnelle répond à une rationalité professionnelle applicable par les équipes.

Ils ne perçoivent pas forcement les bénéfices d'une discussion collective. Leur management essentiellement informel y a rarement recourt. Certains CdS expriment leur désaffection pour les réunions considérées comme des temps improductifs. D'autres sont convaincus de la nécessité des temps d'échange collectif, mais ont renoncé faute d'avoir trouvé la méthode pour dépasser les contraintes organisationnelles : suppression des temps de chevauchement suite à une organisation du travail en 12 heures, priorité donnée à l'activité de soins au détriment des réunions qui ne peuvent avoir de crédit dans le contexte de travail, coût d'une réunion dans un contexte de pénurie de ressources humaines.

Du coté des équipes, les entretiens montrent que celles-ci n'ont pas établi de lien entre le problème et les actions mises en œuvre, même si par ailleurs elles valident bien l'existence du problème à résoudre. A la différence du modèle inter-compréhensif précédent, les discours des professionnels ont été laconiques. Ils ont manifesté un détachement vis-à-vis du projet et une incompréhension, considérés par certains $\mathrm{CdS}$ pour de la résistance, comme en témoigne le PQT 09 (Encadré 2).

Les équipes ont souligné ne pas comprendre la ligne directrice des changements et le sens organisationnel. Elles pointent les limites d'un management informel parfois avec humour et une certaine désinvolture : "Réunion, non, C'est quoi ? [ricanement général] Ben le problème c'est que c'est toujours comme ça, c'est à la va-vite, [...] et il n'y a jamais un moment où il y a une grosse réunion où on parle de tous les problèmes qu'on a vraiment. »

\section{Encadré 2 : Mettre en place la traçabilité de l'entretien pour la certification (PQT 09)}

L'entretien des locaux n'est pas tracé. Pour corriger le problème, le CdS développe différents tableaux de traçabilité dans chacune des pièces, ainsi qu'un tableau général dans l'office. Les tableaux indiquent les périodicités d'entretien : quotidienne, hebdomadaire, mensuelle, trimestrielle et annuelle. Il est demandé aux professionnels chargés de l'entretien de marquer leurs initiales une fois l'entretien réalisé. Mais peu de personnes renseignent les documents. De son côté, le CdS ne comprend pas d'où vient la résistance. Quant aux professionnels, ils ne comprennent pas ce qui leur est demandé. Ils méconnaissent certaines dénominations, comme le terme «cimaise ». De plus, le tableau n'est pas adapté, par exemple il comporte une seule case pour recevoir les 52 initiales de l'entretien hebdomadaire. Ces anomalies organisationnelles ne sont pas remontées directement au CdS par la discussion. C'est le groupe d'étudiants qui a servi de tiers pour permettre au CdS de comprendre qu'il ne s'agissait pas de résistance de l'équipe, mais d'une incompréhension des professionnels et d'une incohérence du document.

La posture prescriptive est identifiée dans quatre PQT (Tableau 2) auprès des médicotechniques (2/4) et infirmiers (2/11). 


\section{Une posture inter-compréhensive asymétrique}

Entre ces deux postures managériales opposées, l'analyse met en évidence une attitude intermédiaire qualifiée de posture inter-compréhensive asymétrique.

Cette posture se traduit par une grande fermeté dans la gestion de la trajectoire du projet. A partir d'une analyse organisationnelle et de certains principes réglementaires et professionnels, ces CdS définissent une nouvelle organisation, ensuite débattue collectivement en réunion. Les discussions conduisent à la formalisation de règles et de procédures, fruits de la négociation. Une fois validée cette organisation est ensuite mise en œuvre et contrôlée de façon stricte par les CdS. Les ajustements sont marginaux en fonction de l'évaluation faite par les $\mathrm{CdS}$.

Les équipes rencontrées ont exprimé leur faible marge de manœuvre, mais elles soulignent surtout la satisfaction de retrouver une organisation collective cohérente, claire, structurée, qui fait fi des arrangements individuels autonomes. Comme l'illustre le PQT 11 (Encadré 3), la définition d'un cadre de travail strict a pour effet de limiter les tensions interpersonnelles, de sécuriser les pratiques et les conditions de travail sans empêcher l'intercompréhension. Au final, CdS et équipes reconnaissent que le contexte nécessitait un management plus directif, non pas dans la perspective d'imposer les orientations au projet, mais pour renforcer la cohésion du collectif de travail.

Encadré 3 : Améliorer les relations de travail dans une équipe pluri-professionnelle (PQT 11)

Le CdS, arrivé dans l'unité depuis trois mois, a rapidement constaté une ambiance de travail particulièrement dégradée avec des retentissements sur la prise en charge des patients et des délations sur les réseaux sociaux. Face à ce problème, le groupe d'étudiants préconise de se rapprocher des psychologues du travail disponibles dans l'institution. Cette stratégie a déjà été tentée dans le passé, avec la mise en œuvre d'un groupe de parole qui a été déserté par les professionnels. Le $\mathrm{CdS}$ préfère réunir l'équipe, poser de façon factuelle les données du problème, rappeler les missions de chacun et la déontologie professionnelle, avant de proposer une nouvelle organisation du travail pour une prise en charge interprofessionnelle coordonnée des patients. Au final ce recadrage collectif par le CdS, accompagné d'une série de mesures pour retravailler ensemble, permet de retrouver une organisation collective. Les professionnels rencontrés témoignent d'une amélioration des conditions de travail. Le CdS est satisfait et dit qu'il aurait fait appel à la médecine du travail si sa démarche avait échoué.

Lors de l'enquête, nous avons rencontré cette posture inter-compréhensive asymétrique dans deux PQT (Tableau 2), chez un CdS médico-technique (1/4) et un CdS infirmier (1/11).

\section{L'implication des responsables hiérarchiques et fonctionnels dans les projets de changement}

Les changements organisationnels portés par les cadres peuvent parfois être dépendants du soutien de certains «acteurs institutionnels »: une autorité hiérarchique, administrative ou médicale (Tableau 2). La modification d'une règle institutionnelle ou l'attribution de ressources ne sont pas à la portée immédiate du $\mathrm{CdS}$, elles dépendent de la validation de ces acteurs institutionnels. Dans ce type de situation, les CdS sont attentifs à identifier les acteurs dont ils dépendent et à anticiper la position qu'ils pourraient adopter face au projet. Ils commencent par chercher les soutiens, tester les engagements, repérer les arguments porteurs pour intéresser leurs interlocuteurs. Cette analyse les conduit aussi à adapter le projet de telle façon qu'il puisse être accepté plus facilement. D'une façon plus générale cette analyse 
préalable à l'action permet de rechercher une plus grande convergence entre les ambitions des $\mathrm{CdS}$ et les attentes des acteurs en position d'autorité. En fonction des appuis que le $\mathrm{CdS}$ parvient à obtenir, deux attitudes se sont distinguées, une intercompréhension basée sur des affinités, ou au contraire la persistance d'un cloisonnement organisationnel.

\section{Une intercompréhension basée sur des relations d'affinité}

Parmi les PQT étudiés (Tableau 2), une partie d'entre eux témoignent d'un bon alignement des objectifs entre les CdS et les acteurs institutionnels. L'enquête n'a pas permis d'identifier des facteurs organisationnels favorables à cet alignement pendant le PQT et pendant le suivi. Néanmoins, elle montre que cet alignement des objectifs autour d'un projet porté par le CdS provient d'un bon niveau d'intercompréhension des logiques de chaque acteur, intercompréhension facilitée par l'existence préalable de relations affinitaires dont la portée dépasse le PQT pris isolément (Encadré 4).

\section{Encadré 4 : Optimiser la coordination professionnelle (PQT 13)}

En réponse à des exigences institutionnelles, le volume de l'activité de l'unité chirurgicale a beaucoup progressé. Cette importante augmentation d'activité nécessite de revoir les organisations de travail, mais elle se traduit par la demande d'une augmentation des ressources humaines. Ces deux axes ont été travaillés collectivement entre le $\mathrm{CdS}$, le cadre coordinateur de pôle et le médecin responsable de l'unité. Le CdS a construit le projet avec chacun en fonction des champs de responsabilités respectives. Le médecin est sollicité pour l'aménagement des locaux en lien avec un responsable des services techniques. L'augmentation des effectifs est calculée avec le cadre coordinateur de pôle et discutée avec la direction des ressources humaines. Les perspectives sont présentées et argumentées au directeur référent qui valide et soutient le projet. La cohérence globale du projet, défendu par tous les responsables a permis de convaincre l'institution et d'obtenir les moyens demandés en un peu moins d'une année.

\section{Une absence d'intercompréhension dans un environnement cloisonné}

En dehors de ces relations affinitaires, la capacité des $\mathrm{CdS}$ à mobiliser les acteurs institutionnels (communauté médicale, administrative, syndicale) semble limitée. Dans plusieurs secteurs, les CdS n'ont pas identifié les conditions institutionnelles nécessaires pour conduire le PQT (Tableau 2). Pour autant, même si les CdS ne trouvent pas d'alliances, ils rencontrent rarement des oppositions formelles ou hostiles aux projets. Les arguments et la logique portés par le $\mathrm{CdS}$ ne trouvent pas d'écho auprès de ces acteurs, principalement parce que les $\mathrm{CdS}$ renoncent à les impliquer formellement dans les projets. Un CdS évoque une «héminégligence » pour désigner sa défaillance à intégrer les acteurs institutionnels plus distants. Les $\mathrm{CdS}$ ont une certaine appréhension à mobiliser de façon formelle ces acteurs institutionnels. Ils estiment ne pas avoir suffisamment de légitimité pour les solliciter. D'ailleurs pour les 11 PQT dont la réalisation dépendait d'acteurs institutionnels extérieurs au service, aucun n'a fait l'objet d'un groupe de travail malgré les souhaits exprimés par certains CdS.

Dans cette situation, les CdS se trouvent démunis pour faire évoluer le PQT de façon collective. Ils expriment des enjeux contradictoires entre, d'un côté leur souhait de poser de façon factuelle, formelle et collective les données du problème à traiter, et de l'autre leur loyauté vis-à-vis de leur unité de soins. Rendre visibles et publics des problèmes internes exposerait davantage les $\mathrm{CdS}$ aux critiques et aux interventions extérieures qui réduiraient d'autant leur marge de manœuvre. 
Face à ce dilemme, les $\mathrm{CdS}$ adoptent deux stratégies alternatives, généralement conduites de façon simultanée. En coulisse, les CdS utilisent l'opportunité de rencontres avec les acteurs institutionnels concernés pour tenter de les impliquer dans le projet de façon informelle. Ces sollicitations sont individuelles, adressées spécifiquement à chaque acteur important. Les CdS tentent d'obtenir des aménagements des positions individuelles de ces acteurs dans la perspective de les articuler ensuite autour du PQT.

En parallèle, lors de rencontres institutionnalisées, certains CdS saisissent ces opportunités pour solliciter le ou les acteurs dont ils sont dépendants. Ces interpellations bénéficient $\mathrm{du}$ caractère formel des échanges pour exercer une influence sur leurs interlocuteurs. Les CdS font alors valoir des arguments légitimes, comme par exemple rappeler les orientations des projets institutionnels. Ces échanges sont aussi une opportunité pour vérifier la détermination des acteurs institutionnels.

Au final, ces interpellations sont restées vaines et sans succès sur l'année de suivi des PQT. Les CdS déclarent à la fois ne pas mettre trop d'espoir dans ces tentatives et en même temps ne jamais renoncer à saisir ces opportunités. Mais ils ne veulent pas non plus qu'on puisse leur reprocher ne pas avoir tout tenté pour améliorer la coordination collective. La gestion des contradictions fait partie d'un quotidien qui les conduit à composer avec cette inertie institutionnelle : "Il y a une telle inertie, que... Peut-être qu'un jour, on va ressentir les effets positifs. Mais là pour le moment, on a fait que donner un coup de barre. [...] On va faire des kilomètres avant que le bateau commence seulement à bouger. Donc c'est ça qui est un petit peu difficile, qui est long, qui est dès fois décourageant. Mais peut-être que ça va porter ses fruits. »

Mais les CdS utilisent aussi la lourdeur bureaucratique, les ambiguïtés et le flou organisationnel pour préserver leurs marges de manœuvre et définir eux-mêmes l'orientation donnée au PQT. Plusieurs CdS ont réussi à clarifier la situation en se détachant de la dépendance institutionnelle. Deux stratégies se distinguent. Certains ont redéfini le périmètre du projet dans une dimension interne à l'équipe pour éviter d'être dépendants de décisions externes. Ils explorent les espaces d'autonomie en marge du contexte institutionnel. Ils ne font pas appel aux appuis institutionnels perçus davantage comme des contraintes et des pertes de temps, que des ressources pour agir. Pour ces CdS, garder la maitrise est synonyme d'efficacité et de réactivité comme en témoigne le PQT 11 présenté précédemment (Encadré 3). D'autres au contraire «jouent la montre » pour éviter de se retrouver en porte-à-faux et hypothéquer leur légitimité managériale face au risque estimé d'un réajustement majeur du projet par les acteurs institutionnels. C'est le motif invoqué par certains $\mathrm{CdS}$, qui ont attendu une clarification institutionnelle pour conduire le projet en proximité (Tableau 2), comme en témoigne le PQT 15 (Encadré 5).

Encadré 5: Conserver l'expertise professionnelle dans le projet institutionnel de mutualiser trois équipes (PQT 15)

Dans le cadre d'un projet institutionnel de réorganisation d'un secteur d'activités, le $\mathrm{CdS}$ a récupéré la responsabilité de trois unités de soins et la consigne de décloisonner les organisations pour améliorer la performance. Le projet, porté et communiqué au niveau institutionnel, n'a fait l'objet d'aucune communication formelle et collective au niveau local : pas de réunion pour définir les nouveaux périmètres des responsabilités des différents acteurs, aucune présentation de la nouvelle organisation. Le CdS sollicite le directeur référent pour qu'une charte de fonctionnement soit rédigée collectivement. Celle-ci est écrite par le directeur et provoque la désapprobation de la communauté médicale au moment de sa diffusion. Dans le contexte de restructuration, tout le monde dit avoir perdu ses repères. Le 
CdS tente une coopération avec les responsables médicaux des deux nouvelles unités attribuées. La réponse frontale du premier conduit le $\mathrm{CdS}$ à se désolidariser du secteur. La faible considération du second, amène le $\mathrm{CdS}$ à redéfinir formellement son rôle dans la nouvelle configuration. Un an après le $\mathrm{CdS}$ gère de façon indépendante les deux unités. La position du cadre coordinateur de pôle n'a pas été évoquée dans le discours du CdS.

La réticence à solliciter des acteurs institutionnels et à élargir le PQT dans cette direction, tient aussi à l'expérience de certains projets participatifs initiés par des acteurs institutionnels. A l'issue de ces démarches participatives, les équipes ont eu le sentiment d'avoir été manipulées. Elles n'ont pas retrouvé dans les décisions une prise en compte de leurs attentes, contraintes ou propositions. Les démarches participatives entrainent de nombreuses attentes et l'institution n'est pas toujours en capacité de les satisfaire. Ce type d'expérience encourage les $\mathrm{CdS}$ à une plus grande prudence dans la sollicitation formelle d'acteurs institutionnels : ils préfèrent garder la maîtrise de leur PQT.

Cette situation a été rencontrée dans deux projets dont le PQT 16 (Encadré 6). Dans ces projets, les CdS ont pu tenir un rôle de porte-parole et de relais entre l'équipe et l'institution. Les soignants impliqués ont eu le sentiment amer d'avoir été utilisés. Un professionnel qualifie la participation de «mascarade ». Les CdS confient leur impuissance. Ils partagent la position de l'équipe, mais ne peuvent marquer leur opposition à une organisation qu'ils sont aujourd'hui contraints de mettre en œuvre. Les CdS ont cherché malgré tout à réduire l'écart entre la logique professionnelle et la logique institutionnelle sans pour autant constater d'évolution sur la période de suivi des PQT.

\section{Encadré 6: Pérenniser une organisation des astreintes sur le volontariat (PQT 16)}

Les soignants, le $\mathrm{CdS}$ et l'équipe médicale ont porté un projet d'astreinte de nuit pour répondre aux besoins de certains patients qui nécessitent une prise en charge répétée sur 24 heures. Après un premier refus, quelque temps plus tard, l'obligation réglementaire conduit à ce que le projet puisse voir le jour au niveau institutionnel. Des professionnels participent à un groupe de travail destiné à définir les modalités d'organisation. Ils ont exprimé leur volonté professionnelle pour une continuité de prise en charge 24/24. Mais la décision institutionnelle est de limiter l'astreinte à minuit, ce qui provoque la colère de l'équipe et son désengagement dans la participation aux astreintes. Les professionnels rencontrés disent avoir fait preuve de naïveté et dénoncent l'hypocrisie d'un système qui, selon eux, cherche simplement à répondre au cadre réglementaire. Ils considèrent porter une part de responsabilité dans cette organisation qui ne répond pas aux besoins. Ils ajoutent qu'il ne fallait plus compter sur eux pour participer aux projets institutionnels.

On trouve des échos de ces difficultés dans les témoignages des équipes. Qu'il y ait succès ou échec du PQT dans cette dimension relationnelle, les entretiens avec les professionnels montrent que les $\mathrm{CdS}$ sont reconnus pour leur soutien aux équipes. Ils sont considérés comme des représentants de l'organisation locale. Les équipes ont bien conscience des contraintes, des tensions entre des logiques différentes et des difficultés auxquelles les $\mathrm{CdS}$ doivent faire face. Dans les situations d'échec, les équipes soulignent les décalages entre les projets institutionnels et la réalité du travail et dédouanent la responsabilité des CdS. 


\section{La conduite du changement en relation transversale}

Les PQT peuvent aussi concerner d'autres services avec lesquels ils interagissent (Tableau 2). L'amélioration de l'organisation d'un service peut dépendre de modifications dans les pratiques d'une autre unité.

Dans les trois PQT qui portent une dimension transversale, les CdS s'appuient sur l'existence d'une procédure institutionnelle. Dans ce contexte, la procédure officielle est une ressource, qui par ailleurs limite la prise en compte des spécificités organisationnelles des secteurs concernés. L'objectif est d'obtenir des autres unités qu'elles se conforment à la procédure, les écarts étant une source importante de dysfonctionnements. Pour autant, les CdS conservent une certaine latitude pour exiger l'application de la règle par les unités concernées. Ils pourraient chercher à faire respecter la procédure telle qu'elle est formulée. Mais dans les cas étudiés, les CdS adoptent plutôt une posture d'intercompréhension dans l'objectif d'engager les unités concernées dans un projet de changement partagé. La stratégie est de systématiquement chercher des ajustements au cas par cas. Ils organisent des réunions avec chacune des unités ciblées, pour comprendre leurs pratiques respectives, notifier les dysfonctionnements et identifier les contraintes réciproques. Les $\mathrm{CdS}$ proposent ensuite des solutions acceptables pour les deux parties. Ils ne cherchent pas à faire respecter à la lettre la procédure formelle existante. Ils préfèrent une convention négociée localement qu'ils savent applicable comme l'illustre le PQT 02 (Encadré 7).

Tous les $\mathrm{CdS}$ ont attribué le succès de leur démarche à leurs relations personnalisées à chaque unité et adaptée aux spécificités cliniques de celles-ci. Un CdS qualifie ce travail par l'expression «prendre sa blouse », qui traduit simultanément l'exploitation de connaissances professionnelles soignantes et la proximité organisationnelle transversale.

\section{Encadré 7: Hiérarchiser la réception des examens de laboratoire à la réception (PQT 02)}

Un nombre très important d'examens arrive avec l'identification d'urgence vitale. Un recueil de données permet d'identifier trois unités dont la pratique est de transmettre en série des examens en urgence. Le $\mathrm{CdS}$ du laboratoire rencontre les $\mathrm{CdS}$ des unités cliniques pour comprendre les logiques. En pratique, il ne s'agit pas d'urgences vitales, mais du besoin des résultats dans un délai de deux heures pour en disposer au moment de la visite médicale ou réaliser des examens programmés. Or avec l'envoi standard des prélèvements les résultats sont garantis dans un délai d'une heure. Mais pour rassurer les unités, le CdS propose qu'un papier rouge soit ajouté dans la pochette pour que l'examen soit traité en priorité, sans pour autant être considéré comme une urgence vitale. Cet arrangement convient à tous et permet de normaliser la situation. Le CdS projette un retour à la procédure standard une fois que les unités auront confiance dans le processus. Le papier rouge disparaitra de lui-même.

Les postures déclarées par les CdS sont confirmées par les membres des équipes qui ont conscience du rôle transversal joué par leur CdS. Il est reconnu comme un acteur pivot pour réduire les dysfonctionnements organisationnels et ainsi améliorer les conditions de travail des équipes.

\section{Discussion}

Quel que soit le type d'acteurs que le CdS tente de mobiliser, le succès du PQT dépend de la capacité du CdS à exercer un leadership fondé sur un rôle d'animation et une attitude d'intercompréhension. L'équipe le considère alors comme un pivot organisationnel dont l'autorité est reconnue et valorisée. Il est considéré par l'équipe et les partenaires transversaux 
comme un représentant légitime pour négocier des arrangements avec les autres unités. A l'inverse, des démarches plus directives, inspirées par certaines rationalités professionnelles et exclusives des autres rationalités concernées, ont été génératrices d'incertitudes, d'incompréhensions et de tensions.

Ce premier constat mérite deux nuances. Tout d'abord, une gestion trop informelle du PQT, sans espace de discussion organisé, entraine les mêmes phénomènes d'incertitude et de tension. D'autre part, les capacités d'action du CdS restent limitées dès lors que les PQT impliquent des décisions institutionnelles, compte tenu des difficultés qu'il rencontre à engager des acteurs plus distants. Mais cette limitation ne se traduit pas par une perte d'autorité auprès des équipes, bien conscientes des difficultés que les $\mathrm{CdS}$ rencontrent.

\section{Avantages et limites de l'étude}

Le dispositif pédagogique a constitué une opportunité pour comprendre la posture du $\mathrm{CdS}$ dans les mécanismes de transformation organisationnelle. L'étude a permis d'explorer la fonction de pivot dans différentes dimensions managériales.

La triangulation des 17 discours des CdS et des professionnels a permis de valider les données recueillies. Pour autant il aurait été judicieux d'étendre le terrain d'enquête à tous les protagonistes impliqués dans les deux autres dimensions relationnelles analysées, en particulier pour les personnels administratifs et médicaux. Un recueil de données supplémentaire aurait permis de compléter l'analyse et de mieux cerner les motifs de tension qui sont sources de cloisonnement. Malgré tout les résultats viennent compléter les connaissances existantes de la fonction CdS et sur les organisations hospitalières.

La discussion des résultats se structure autour de la diversité des modalités d'exercice de l'autorité du CdS, avec trois contributions. Cette autorité s'appuie sur une expertise soignante et une compétence managériale vis-à-vis de son équipe, mais aussi des relations affinitaires avec les acteurs extérieurs. En fonction de la place accordée à l'expertise soignante, l'exercice de l'autorité peut conduire à un management directif involontaire. Pour finir, l'autorité du CdS suppose la préservation d'une autonomie pour conduire le projet.

\section{Une pratique managériale qui articule les valeurs du soin et les enjeux gestionnaires}

Différents travaux sur le manager de proximité tendent à montrer que le cadre n'arrive pas à prendre sa place dans les activités de coordination organisationnelle. Plusieurs auteurs montrent que le CdS est pris dans des conflits de rôle (20) le partageant entre une logique métier, un attachement aux valeurs soignantes $(22,28,35)$, et une logique managériale $(27,42)$. Il n'est jamais à la bonne distance entre une proximité, qui le conduit à ce que certaines dimensions de son rôle lui échappent (27), ou à l'inverse pris dans une aspiration gestionnaire (14) qui le déconnecte des préoccupations de terrain.

Or nos résultats montrent que le CdS arrive à tenir une position intermédiaire qui combine ces deux dimensions. Sa connaissance fine de l'organisation et des soins est un atout pour définir et travailler des problèmes reconnus comme pertinents par les équipes (9). La volonté du $\mathrm{CdS}$ à résoudre ces problèmes confirme son attachement aux valeurs du soin. Mais en définissant ces problèmes, il assure en même temps sa mission de transformation de l'organisation dans l'objectif d'une performance organisationnelle. De plus le développement des PQT dans les trois directions relationnelles montre qu'il ne se cantonne pas exclusivement à une gestion en proximité, mais qu'il est bien un pivot organisationnel (1-3). 


\section{La place de l'expertise professionnelle dans l'exercice de l'autorité}

Même si le CdS définit des problèmes organisationnels qui sont la préoccupation des équipes, il est nécessaire de s'arrêter sur la manière dont le projet est géré. Qu'est-ce qui motive le $\mathrm{CdS}$ à développer un mode managérial inter-compréhensif ou au contraire prescriptif?

Tout en étant très prudent sur l'interprétation des résultats, ceux-ci montrent malgré tout une catégorisation en fonction des origines professionnelles des CdS. Sur l'échantillon, les infirmiers se distribuent sur les deux modalités alors que les médicotechniques sont davantage prescriptifs et les rééducateurs inter-compréhensifs. Cette hypothèse est apparue suite à la réflexion d'un $\mathrm{CdS}$ rééducateur qui a comparé son activité professionnelle à celle du manager. Un des principes professionnels en rééducation est de toujours travailler avec le patient, aller contre sa volonté n'étant jamais productif. Ce parallèle retrouvé dans les travaux d'Hesbeen (35) montre que la volonté du CdS ne suffit pas et que la capacité de mobilisation passe par une prise de conscience de la nécessaire considération de l'équipe. Entre vouloir, pouvoir et savoir changer l'organisation, Nobre (3) rappelle que la transformation ne se décrète pas, elle s'accompagne. Les Travaux d'Edmonson (34) et Habib (9) confirment ce principe, comme Detchessahar et al. qui stipulent que cet accompagnement doit passer par des espaces de discussion sur le travail $(14,36)$.

En distinguant les cultures professionnelles, c'est en réalité la place de la temporalité qui est interrogée. Si on poursuit la métaphore de la rééducation, il existe une différence entre la prise en charge sur le long terme et le soin aigu, technique sur le court terme. Les travaux sur la population $\mathrm{CdS}$ décrivent une communauté issue du métier d'infirmier qui privilégie la réactivité (42), soumise à une pression d'immédiateté (14). Cette pression rend le découplage temporel difficile (9). Or, les démarches inter-compréhensives nécessitent des délais de conception et de mise en œuvre plus longs que les démarches prescriptives, a priori plus rapides.

La comparaison des modes managériaux inter-compréhensifs versus prescriptifs permet d'identifier deux effets délétères pour la conduite du changement. La première cause est liée à un management informel qui conduit secondairement le $\mathrm{CdS}$ à réfléchir à la place de l'équipe.

Dans plusieurs PQT, l'activité de coordination organisationnelle s'est traitée de façon informelle et invisible $(11,43)$, sur le même mode opératoire que la coordination opérationnelle $(4,12)$. Cette production au fil de l'eau constitue une première limite pour développer une prise de distance et une réflexion collective (31). Cette logique d'action réactive conduit à une démarche prescriptive des changements organisationnels au-delà même de la volonté du $\mathrm{CdS}$ qui cherche simplement à résoudre le problème au plus vite. La réactivité supprime les temps pour interroger l'équipe et favoriser sa participation (9).

La seconde conséquence découle en partie de la première. Faute d'échanges collectifs, le $\mathrm{CdS}$ réfléchit à la place de l'équipe et de ce fait impose sa rationalité professionnelle. Au final, dans ce schéma prescriptif, l'équipe est court-circuitée, laissée en marge de la conduite du changement (44). Pour autant, le CdS ne veut pas imposer son autorité, même si au final c'est celle-ci qui s'impose. Il cherche avant tout à aider et faciliter le travail de l'équipe en lui apportant les solutions au problème. Malgré cette intention louable, la démarche ne permet pas de susciter l'intérêt et l'engagement nécessaires pour favoriser la transformation organisationnelle. Au final la stratégie est contreproductive car elle produit l'incompréhension des deux parties. Les propos des professionnels confirment les conclusions de différents travaux de recherche $(14,15,36)$ : les équipes souffrent davantage d'un manque de management que d'un excès. 
A l'inverse, si les connaissances des professionnels sont utilisées par le CdS pour animer les débats avec l'équipe sur les perspectives organisationnelles, les échanges alimentent la réflexion de l'équipe, son autonomie et sa responsabilisation $(9,27)$. Cette intercompréhension produit également de la reconnaissance professionnelle qui favorise l'engagement des acteurs et donne du sens à l'objectif de transformation. L'animation d'espaces de discussion favorise la conduite du changement organisationnel $(14,15,36)$.

Les CdS intègrent tous, dans leur raisonnement, leurs savoirs professionnels. Leur légitimité managériale dépend de cette combinaison entre connaissances des pratiques et des organisations de soins. Mais encore fallait-il que ces savoirs soient utilisés à bon escient. Affirmer sa connaissance professionnelle peut ainsi être plus délétère que constructif pour accompagner le changement des organisations.

\section{Une autonomie préservée vis-à-vis des contraintes institutionnelles}

Dans les organisations hospitalières, la difficulté pour coordonner des logiques différentes n'est pas de trouver des professionnels aux compétences diversifiées, comme pour la gestion de grands projets industriels (45). La difficulté est de réunir ces compétences diversifiées aux enjeux différents dans un environnement qui laisse une grande autonomie aux acteurs $(6,11)$. A la différence d'autres projets dont l'objectif est de trouver des solutions pour réduire les incertitudes organisationnelles, ici les solutions sont déjà identifiées. La difficulté est d'arriver à coordonner des enjeux stratégiques diversifiés autour d'un axe qui permette la convergence. Mais l'absence d'espace de travail formel et collectif entre les acteurs institutionnels illustre la force des cloisonnements et des autonomies respectives (46). Les $\mathrm{CdS}$ peuvent aussi alimenter ces tensions alors que d'autres tentent de construire par des relations individuelles dans des espaces informels. Mais ces couplages ne permettent pas la coordination collective et restent fragiles. Ils dépendent des personnes au gré des affinités, mais pas des fonctions entre les responsables hiérarchiques, administratifs ou médicaux. Nos résultats ne mettent pas en évidence une intercompréhension plus facile avec une catégorie plutôt qu'une autre, contrairement à d'autres travaux (28). De même, le CdS n'est pas non plus soumis à l'autorité médicale, contrairement à ce que défend Mucchielli (26). En revanche on constate comme Burelier (28) que le CdS développe des stratégies en marge des injonctions qu'il reçoit qui lui permettent de développer son pouvoir d'agir.

Le CdS n'a pas le leadership pour monter un groupe de travail inter-logique et convoquer les protagonistes impliqués pour travailler collectivement les problèmes. Il est un acteur parmi d'autres, autres dont l'autorité est généralement supérieure à la sienne. Ainsi pour conduire le changement dans la dimension institutionnelle, le $\mathrm{CdS}$ au leadership opérationnel doit trouver le soutien institutionnel de celui qui a le leadership politique (1), sinon rien de visible ne se réalise.

La difficulté à faire progresser le PQT dans la dimension institutionnelle pendant l'année de suivi montre les limites pour rendre plus perméable les cloisonnements $(7,9)$. Il est difficile de négocier des consensus collectifs $(11,13)$ qui conduiraient à rendre formels des conflits de valeurs (26). La gestion informelle du PQT, à partir de relations interpersonnelles, permet au CdS de nuancer les positions contradictoires et d'orienter le projet.

Compte tenu de sa position hiérarchique, le CdS ne cherche pas à s'engager de façon formelle dans l'espace institutionnel. Le CdS est un traducteur invisible. Il préfère être un négociateur de l'ombre, à distance de l'espace institutionnel et de ses contradictions. Cette posture lui permettra de préserver son autonomie et de limiter son exposition dans des luttes institutionnelles incertaines. Même si les enjeux sont d'une autre nature, cette attitude est 
somme toute cohérente avec celle qu'il adopte pour la coordination opérationnelle décrite comme un «travail de lien invisible » (4).

Il utilise les flous et la complexité organisationnelle pour se frayer une trajectoire autonome en délimitant lui-même le périmètre du projet, même si cette autonomie est utilisée dans l'objectif d'améliorer l'efficacité organisationnelle (2). Ainsi la posture du CdS identifiée dans le suivi des PQT traduit certaines formes de résistances $(2,10)$. Il n'applique pas à la lettre, il n'a pas la fonction de «courroie de transmission », mais plutôt celle de «tiers » (47) qui adapte les contraintes institutionnelles (8) et dans certaines situations aménage les injonctions qui lui sont faites $(44,48)$.

Il développe un système relationnel individualisé, plutôt que de chercher à mettre autour de la table toutes les tensions de logiques différentes. Car malgré une certaine volonté affichée du CdS à vouloir réduire les ambiguïtés, en pratique chacun tire les bénéfices d'une situation insatisfaisante mais stable. La rationalité affichée par les acteurs institutionnels est préservée (19), les logiques catégorielles ne sont pas remises en cause (42) et les antagonismes connus ne sont pas officialisés (36). De plus, la gestion informelle permet au CdS de préserver sa loyauté professionnelle (49). Elle lui évite de réactiver les ambiguïtés de rôle entre logique de soin et de gestion (20) et de porter la responsabilité du risque d'échec (5).

Si les résultats montrent l'inertie d'un système qui se modifie à la marge en fonction des enjeux et des affinités dans une gestion informelle, ils permettent aussi d'identifier les effets d'une gestion institutionnelle plus formelle. Dans certains secteurs, les acteurs ont fait référence à d'anciens groupes de travail institutionnels et décrit des situations de blocage. Les $\mathrm{CdS}$ se retrouvent alors en porte-à-faux entre deux mondes qui n'ont pu se concilier, le monde soignant avec ses valeurs professionnelles et le monde de l'administration avec ses logiques de gestion $(8,9)$. Les démarches participatives ne sont pas toujours suffisantes pour réduire les écarts (10). Le travail collectif ne permet pas systématiquement de constituer un objet unique par l'articulation et l'alignement des visées professionnelles, gestionnaires et politiques $(8,9)$. A défaut, chacun reste sur sa logique et sa rationalité. Le projet perd de sa cohérence, la symétrie relationnelle disparait et le pouvoir institutionnel reprend une place dominante. Non seulement les professionnels ont le sentiment que leur engagement n'est pas récompensé mais, au contraire qu'ils ont été trahis $(9,10,15,19)$. Au-delà du projet lui-même, c'est la confiance envers l'institution qui est altérée sur le long terme. Ces expériences traduisent que la démarche participative ne représente pas la condition du succès. L'unique regroupement d'acteurs ne fait pas intercompréhension. C'est l'écoute, la considération et la confiance réciproques qui permettent de trouver un consensus où l'intérêt de chacune des parties est respecté $(9,11,32,44)$.

\section{Recommandations}

Cette exploration de la capacité d'action du CdS dans l'activité de coordination collective permet de formuler quelques recommandations managériales et pédagogiques.

\section{Recommandations managériales}

Pour beaucoup de CdS, il est difficile de s'affranchir de la coordination opérationnelle pour porter des projets organisationnels plus structurés. Pourtant, l'enquête montre que ces projets structurés parviennent à améliorer l'organisation, bien au-delà des efforts quotidiens isolés. Elle montre tout l'intérêt d'organiser des espaces d'échanges sur le travail et de contractualiser davantage les projets d'amélioration à l'initiative des CdS. La coordination 
organisationnelle dépend conjointement de la convergence des intérêts divergents et de l'institutionnalisation de l'action collective (46).

\section{Instaurer des temps d'échanges réguliers sur l'organisation et l'activité de travail}

De nombreux travaux montrent que les équipes souffrent davantage d'une absence de management que d'un excès et soulignent le besoin d'une coordination et d'un management de proximité $(14,15,36)$. Nos résultats confirment que le management doit favoriser l'intercompréhension. Cela nécessite de consacrer du temps dans un espace de travail dédié et distinct du flot quotidien de l'activité (36).

Dans cet espace de discussion, le CdS n'est plus le sachant (22,35), mais l'animateur d'un débat où les savoirs sont distribués. Il cherche à développer une posture qui valorise les compétences de chacun. Il écoute, anime et participe aux débats. Il argumente sa position dans une relation symétrique, sans imposer sa vision du monde. Cette communication favorise la réflexion professionnelle, les apprentissages organisationnels (47) et participe à la reconnaissance individuelle et collective. Il ne s'agit pas pour le $\mathrm{CdS}$ de renier ses savoirs professionnels, mais de favoriser et valoriser l'expression de ceux de l'équipe. Car quand il s'agit de prendre la décision, c'est bien au CdS de peser le pour et le contre pour une coordination qui doit correspondre aux exigences professionnelles.

Certains invoquent les difficultés à instaurer ces temps d'échanges collectifs dans un environnement contraint par la production de soins. Certains CdS tentent de contourner cette contrainte en développant l'animation à intervalles courts. La répétition de mini-temps collectifs contribue à la coordination sous une autre forme que la réunion traditionnelle. Mais il manque le recul suffisant pour valider cette méthode pour conduire des projets organisationnels de la nature des PQT.

D'autres refusent ces débats, considérant ces temps improductifs ou par crainte de se voir débordés par des demandes auxquelles les CdS ne pourraient faire face. En réalité il s'agit de temps de travail très productifs où se règlent les problèmes et se définissent les organisations. Les équipes sont capables de s'engager, uniquement parce que ce qui leur est proposé est digne d'intérêt et porteur de sens, même si la charge de travail est importante (44). Favoriser les dynamiques collectives permet de faire converger les positions, développer de la reconnaissance mutuelle (31) et la satisfaction professionnelle et managériale (15). Elle favorise l'engagement, l'autonomie professionnelle et la responsabilisation (31).

\section{Contractualiser les projets organisationnels}

La coopération implique les protagonistes dans toutes les dimensions relationnelles. Le CdS est identifié comme un facilitateur de coopérations interprofessionnelles qui, pour autant, a besoin du soutien institutionnel pour conduire son action (1). Améliorer la conduite du changement passe par une mise à l'agenda et des actions de communication pour identifier le projet comme un axe de travail spécifique. Cette communication concerne les trois dimensions relationnelles.

Dans un premier temps, l'intention du CdS gagnerait à être explicitée avec son supérieur hiérarchique. L'entretien annuel de progrès peut être l'opportunité d'échanger et de fixer les axes de travail spécifiques. Cette formalisation offre au moins deux avantages. D'une part le projet est posé comme une activité à moyen terme, avec un autre statut par rapport à l'activité opérationnelle. Cela devrait faciliter le découplage avec les pratiques réactives de certains CdS. D'autre part cette contractualisation au niveau hiérarchique favorise l'enrôlement et le soutien institutionnel en faveur d'un couplage avec les autres protagonistes dont le projet 
dépend. La formalisation du projet clarifie les attributions et les responsabilités (26) et facilite les couplages collectifs. Pour aller plus loin, une lettre de mission permet de marquer le soutien au projet et légitimer le $\mathrm{CdS}$ dans cette activité. Par la suite, le rapport d'activité annuel est un outil qui rend compte de l'avancée du projet jusqu'à son terme. Cette visibilité permet de tenir l'engagement des acteurs sur le long terme (15), de reconnaitre l'investissement collectif, le travail accompli $(7,15)$ et contribue aux apprentissages collectifs (3).

La contractualisation de projets organisationnels peut aussi s'adosser à certains dispositifs existants. Par exemple avec les Comités de Retour d'Expérience (CREX) qui traitent des dysfonctionnements d'organisation des soins en groupes pluri-professionnels. Les réunions mensuelles instaurent des espaces d'échanges collectifs ritualisés. L'évènement indésirable est le point d'entrée qui incite l'ensemble des acteurs à unir les réflexions pour comprendre la défaillance au-delà des logiques rationnelles cloisonnées dans un esprit d'intercompréhension (21).

\section{Recommandations pour la formation en management}

Cette recherche managériale sur la population $\mathrm{CdS}$ constitue un support de réflexion pour la formation des étudiants cadres. La transformation organisationnelle est la conjonction équilibrée entre trois facteurs, l'intention (vouloir), la connaissance (savoir) et la capacité d'action (pouvoir). Le premier message délivré aux étudiants le jour de l'entrée en formation cherche à mettre une distance avec l'exercice soignant : "Etre cadre de santé c'est faire le deuil du soin. » Certains étudiants désapprouvent ce qu'ils perçoivent comme le reniement d'une culture soignante au bénéfice de principes gestionnaires. Après les échanges suscités, ce message est complété : «Mais faire le deuil s'est se souvenir. » Les étudiants, comme les $\mathrm{CdS}$, sont animés par les valeurs du soin (10). Être cadre de santé c'est vouloir soigner autrement en rendant les organisations de soins plus performantes. Mais cet engagement ne doit pas être source d'une rationalité professionnelle cloisonnée. Le CdS est un traducteur qui articule des logiques professionnelles différentes. Il doit lui-même prendre de la distance avec le soin pour faciliter l'expression des soignants.

\section{Perspectives scientifiques}

Cet article a présenté le travail des CdS dans l'activité de coordination organisationnelle. Le dispositif pédagogique constituait une opportunité pour observer les dynamiques de transformations organisationnelles. Pour compléter l'exploitation du matériau recueilli, il reste à identifier l'impact du groupe d'étudiants comme tiers dans la conduite de projet. La littérature identifie le tiers externe comme un vecteur pour développer la réflexion individuelle et collective, le pouvoir d'agir et les apprentissages organisationnels (25) dont l'effet reste à déterminer.

\section{Conclusion}

Les convictions professionnelles sont parfois si fortes et idéalisées, qualifiées d'héroïques par Dujarier (10), que de nombreux CdS et prétendants à la fonction ont l'habitude de commencer leur exposé par la formule «En tant que cadre, garant de la qualité des soins ... ». Cette formule est dangereuse, car elle survalorise la responsabilité du manager (50). Un seul acteur, quel qu'il soit, ne peut ni se considérer comme l'unique garant de la coordination $(11,35)$. Se positionner comme "garant », c'est envoyer le message aux autres protagonistes que leur engagement n'est pas nécessaire. Améliorer la coordination c'est au contraire chercher à distribuer les rôles, valoriser les responsabilités, dans un tout qui alors peut être 
coordonné. Il s'agit de redonner de la capacité d'action aux acteurs pour se libérer des entraves d'un système cloisonné à l'opérationnalité collective limitée $(3,6,9,11)$. Le rôle du $\mathrm{CdS}$ dans ce système n'est pas de fermer les perspectives, mais au contraire de les ouvrir $(44,48)$ en cherchant à développer une réflexion sur les pratiques et les apprentissages organisationnels.

\section{Remerciements}

Nous remercions l'ensemble des cadres de santé et professionnels dont la participation et la confiance ont rendu possible ce projet de recherche. Merci aux étudiants de l'IFCS du CHU Grenoble Alpes pour la qualité du travail fourni et l'aide apportée aux CdS pour résoudre des problèmes récurrents d'organisation des soins.

Enfin nous remercions mesdames Geraldine CHEVRON-GABORIAUD et Sylvette MARTINEZ, toutes deux cadres de santé au CHUGA, pour leur relecture et leurs remarques.

\section{Financement}

Ce projet a été financé par le Ministère de la Santé dans le cadre d'un Programme Hospitalier de Recherche Infirmière et Paramédical (PHRIP 2011, ${ }^{\circ}{ }^{26}$ )

\section{Conflits d'intérêts}

Les auteurs ne déclarent aucun conflit d'intérêt.

\section{Bibliographie}

1. François P, Pomey M-P. Implantation de la gestion de la qualité dans les hôpitaux français, jeux d'acteurs et transformations induites. Rev Dépidémiologie Santé Publique. sept 2005;53:1S4-1S11.

2. Desmarais C, Abord de Chatillon E. Le rôle de traduction du manager. Rev Fr Gest. 25 août 2010;(205):71-88.

3. Nobre T. Désapprendre ce qui n'a pas été appris. Rev Int Psychosociologie Gest Comport Organ. Spring 2013;19(47):151-163.

4. Bourret P. Les cadres de santé à l'hôpital: un travail de lien invisible. Paris: Seli Arslan; 2006. $288 \mathrm{p}$.

5. Mintzberg H. Manager : Ce que font vraiment les managers. Paris: Vuibert; 2011. 352 p.

6. Glouberman S, Mintzberg H. Managing the Care of Health and the Cure of DiseasePart I : Differentiation. Health Care Manage Rev. winter 2001;26(1):58-71.

7. Herreros G, Milly B. La qualité à l'hôpital, un regard sociologique. Paris: Editions L'Harmattan; 2011. 152 p. (Conception et Dynamique des Organisations).

8. Grenier C, Guitton-Philippe S. La question des regroupements/mutualisations dans le champ sanitaire et social: l'institutionnalisation d'un mouvement stratégique? Manag Avenir. sept 2011;7(47):98-113. 
9. Habib J, Vandangeon-Derumez I. Le rôle du leader formel dans la transformation des organisations pluralistes: analyse comparée de deux hôpitaux. Rev Fr Gest. mars 2015;(2):45-66.

10. Dujarier M-A. L’idéal au travail. Paris: Presses Universitaires de France; 2012. 237 p.

11. Denis J-L, Langley A, Cazale L. Peut-on transformer les anarchies organisées? Leadership et changement radical dans un hôpital. Ruptures Rev Transdiscipl En Santé. 1995;2(2):165-189.

12. Grosjean M, Lacoste M. Communication et intelligence collective: le travail à l'hôpital. Presses Universitaires de France; 1999. 225 p. (Le travail humain).

13. Mucchielli A. Étude des communications : approche systémique dans les organisations. Paris: Armand Colin; 2004. 160 p. (Collection U).

14. Detchessahar M, Grevin A. Un organisme de santé... malade de «gestionnite ». Ann Mines-Gérer Compr. déc 2009;98(4):27-37.

15. Grevin A. Les transformations du management des établissements de santé et leur impact sur la santé au travail: l'enjeu de la reconnaissance des dynamiques de don. Étude d'un centre de soins de suite et d'une clinique privée malades de «gestionnite » [Internet]. Université de Nantes; 2011 [cité 12 mars 2016]. Disponible sur: https://tel.archives-ouvertes.fr/tel-00683586/document

16. François P, Boyer L, Weil G. Implantation d'une démarche qualité dans les services médicaux d'un hôpital universitaire: facteurs d'accélération et de frein. Rev DÉpidémiologie Santé Publique. juill 2008;56(4):S189-S195.

17. Acker D, Bensadon A-C, Legrand P, Mounier C. Management et efficience hospitalière: une évaluation des déterminants [Internet]. Paris: Inspection générale des affaires sociales; 2012 avr [cité 18 août 2016] p. 111. Report No.: RM2012-046P. Disponible sur:

http://www.robertholcman.net/public/documents_institutionnels/Rapports_IGAS/rapport _igas_management_efficience_hopital_determinants_2012.pdf

18. La HAS déploie PACTE, un programme autour du travail en équipe [Internet]. Haute Autorité de Santé. 2013 [cité 6 sept 2016]. Disponible sur: http://www.hassante.fr/portail/jcms/c_1601344/fr/la-has-deploie-pacte-un-programme-autour-dutravail-en-equipe

19. Hees M. Le management à l'hôpital. Science, art, magie ? 2ème. Paris: Seli Arslan; 2010. 186 p. (Perspective soignante).

20. Dumas M, Ruiller C. «Être cadre de santé de proximité à l'hôpital », quels rôles à tenir ? Rev Gest Ressour Hum. 1 mars 2013;87(1):42-58.

21. François P, Sellier E, Imburchia F, Mallaret M-R. Le comité de retour d'expérience (CREX): une méthode pour l'amélioration de la sécurité des soins. Rev DÉpidémiologie Santé Publique. avr 2013;61(2):155-161. 
22. Abel P-C. Le cadre infirmier de proximité : soignant ou manager?: Identité et perspectives du cadre de proximité au regard d'une éthique du soin. Paris: VuibertEstem; 2014. 128 p. (Sciences du soin).

23. Dujardin P-P, Reverdy T, Valette A, François P. Cadre de santé et gestion de projet d'amélioration de la qualité: analyse de l'action managériale à partir d'une formationaction à la gestion de projet. Rech Soins Infirm. juin 2016;2(125):46-60.

24. Friedberg E. Le pouvoir et la règle. Paris: Seuil; 1993. 329 p. (Points Essais).

25. Volckrick É. Intervenir en tiers aujourd'hui. Négociations. janv 2007;1(7):75-88.

26. Mucchielli A. Soigner l'hôpital: une approche systémique et communicationnelle. In: Les organisations : Etat des savoirs. 2ème. Auxerre: Sciences Humaines; 2005. p. 383389.

27. Buscatto M. Des managers à la marge: la stigmatisation d'une hiérarchie intermédiaire. Rev Fr Sociol. Winter 2002;43(1):73-98.

28. Burelier F. L'hybridation de rôle dans les hôpitaux publics: le compromis identitaire desprofessionnels-managers. In: Défis des organisations de santé : défis des sciences de gestion. Lyon; 2008.

29. Strauss AL. La trame de la négociation: sociologie qualitative et interactionnisme. Paris: Editions L'Harmattan; 1992. 311 p. (Logiques sociales).

30. Akrich M, Callon M, Latour B. A quoi tient le succès des innovations ? 1: L'art de l'intéressement. Gérer Compr Ann Mines. juin 1988;(11):4-17.

31. Monteil P-O. Du management du soin au soin du management. Rev Fr Déthique Appliquée. Winter 2016;(1):71-79.

32. Callon M. Éléments pour une sociologie de la traduction: la domestication des coquilles Saint-Jacques et des marins-pêcheurs dans la baie de Saint-Brieuc. Année Sociol 19401948-. 1 janv 1986;36:169-208.

33. Akrich M, Callon M, Latour B. A quoi tient le succès des innovations ? 2: Le choix des porte-parole. Gérer Compr Ann Mines. sept 1988;(12):14-29.

34. Edmondson AC, Bohmer RM, Pisano GP. Disrupted routines: Team learning and new technology implementation in hospitals. Adm Sci Q. déc 2001;46(4):685-716.

35. Hesbeen W. Cadre de santé de proximité - Un métier au coeur du soin. Issy-lesMoulineaux: Elsevier Masson; 2011. 155 p.

36. Detchessahar M. L'avènement de l'entreprise communicationnelle. Rev Fr Gest. 1 févr 2003;1(142):65-84.

37. Lépine V. La reconnaissance comme condition de la collaboration au sein des unités de soins: les cadres de santé entre intuition et raison. In: Les cadres de santé et la reconnaissance au travail, une position difficile entre soin et management. RueilMalmaison: Editions Lamarre; 2012. p. 121-37. (Bourgeon D. Fonction cadre de santé). 
38. Dujardin P-P, Raynal V, Molinaro A, Vissac C, Capron D, Cholez C. Conduite de changement pour préparer l'avenir. Soins Cadres. août 2011;20(79):38-40.

39. Gioia DA, Corley KG, Hamilton AL. Seeking qualitative rigor in inductive research: Notes on the Gioia methodology. Organ Res Methods. 24 juill 2012;16(1):15-31.

40. Glaser BG, Strauss AL. Discovery of Grounded Theory: Strategies for Qualitative Research. New Brunswick: AldineTransaction; 1967. 271 p.

41. Bardin L. L'analyse de contenu. 2e édition. Presses Universitaires de France; 2013. 320 p. (Quadrige).

42. Coulon R. Le cadre de santé entre logiques d'utilité et logiques de soins hospitaliers [Internet]. Dijon: Université de Bourgogne-CREGO EA7317 Centre de recherches en gestion des organisations; 2010 avr [cité 12 mars 2016] p. 26. (Cahier de recherche du FARGO). Report No.: 1100401. Disponible sur: http://leg.ubourgogne.fr/wp/1100401.pdf

43. Dujardin P-P, Reverdy T, Valette A, François P. Conduite de projet, gestion du collectif de travail et unités de soins. Soins Cadres. mai 2015;24(94):26-8.

44. Mispelblom-Beyer F. Encadrer, un métier impossible? 2e édition. Paris: Armand Colin; 2010. 320 p.

45. Midler C. L'auto qui n'existait pas: management des projets et transformation de l'entreprise. Paris: Dunod; 2012. 262 p. (Stratégie et management).

46. Xhauflair V, Pichault F, Maesschalck MF. Partenariats inter-organisationnels et nouvelles formes de gouvernance : les conditions d'un compromis équilibré et pérenne. Manag Avenir. 12 juin 2010;(33):298-316.

47. Volckrick M-E. Les usages du tiers dans la négociation. Négociations. juill 2009;2(12):131-46.

48. Falcoz C, Laroche H, Cadin L, Frery F. Cet obscur objet du management, Le métier de cadre tel que le voient les diplômés d'une grande école de Commerce. In: Ce que font les cadres [Internet]. Lyon; 2004 [cité 14 avr 2016]. p. 35-48. Disponible sur: http://gdrcadres.cnrs.fr/cahier/Cahier6.pdf\#page $=37$

49. Lépine V. Choisir d'être manager dans l'hôpital public ou l'histoire d'une loyauté mise à l'épreuve. In: Les cadres de santé et la reconnaissance au travail, une position difficile entre soin et management. Rueil-Malmaison: Editions Lamarre; 2012. p. 211-25. (Bourgeon D. Fonction cadre de santé).

50. Vidaillet B, Vignon C. Comment tenir compte de la subjectivité du manager en formation? Ann Mines - Gérer Compr. juin 2009;2(96):62-74. 


\begin{tabular}{|c|c|c|c|}
\hline Thèmes & Catégories & Codes & Relations fréquentes \\
\hline \multirow[t]{2}{*}{$\mathrm{CdS}$} & C06-Difficulté & 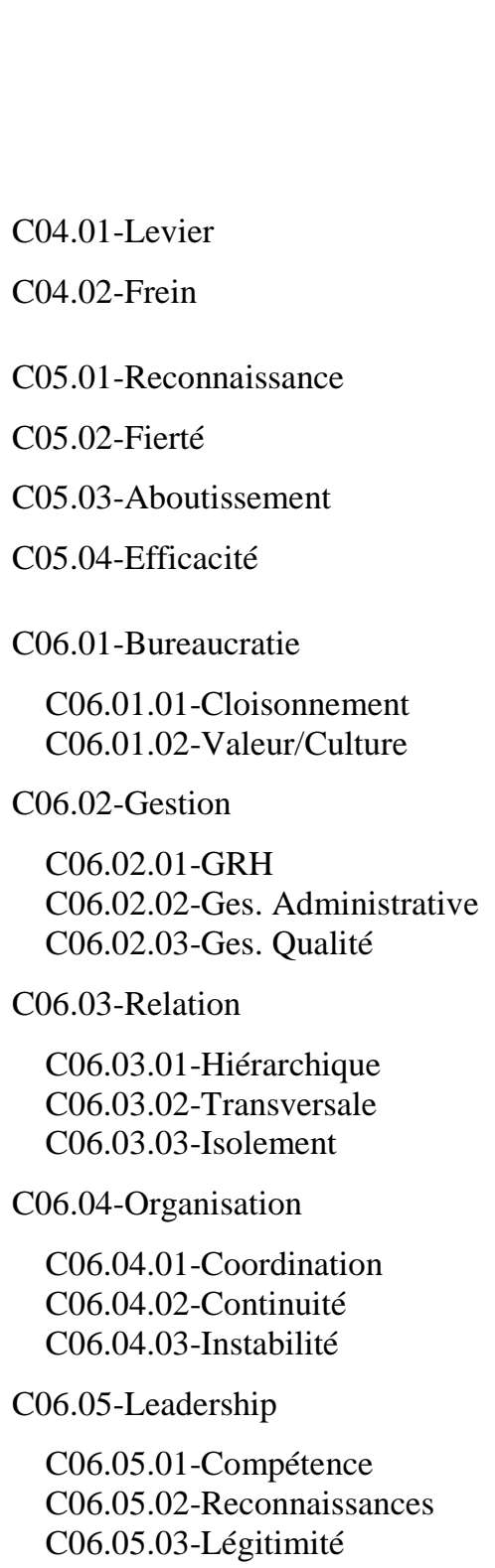 & \\
\hline & $\begin{array}{l}\text { C07-Attente } \\
\text { C08-Style management } \\
\text { C09-Organisation }\end{array}$ & $\begin{array}{l}\text { C08.01-Participatif } \\
\text { C08.02-Délégatif } \\
\text { C08.03-Directif } \\
\text { C09.01-Orga. Formelle } \\
\text { C09.02-Orga. Informelle } \\
\text { C10.01-Com. Formelle } \\
\text { C10.02-Com. Informelle }\end{array}$ & \\
\hline Soignants \& & S01-Analyse & & \\
\hline
\end{tabular}




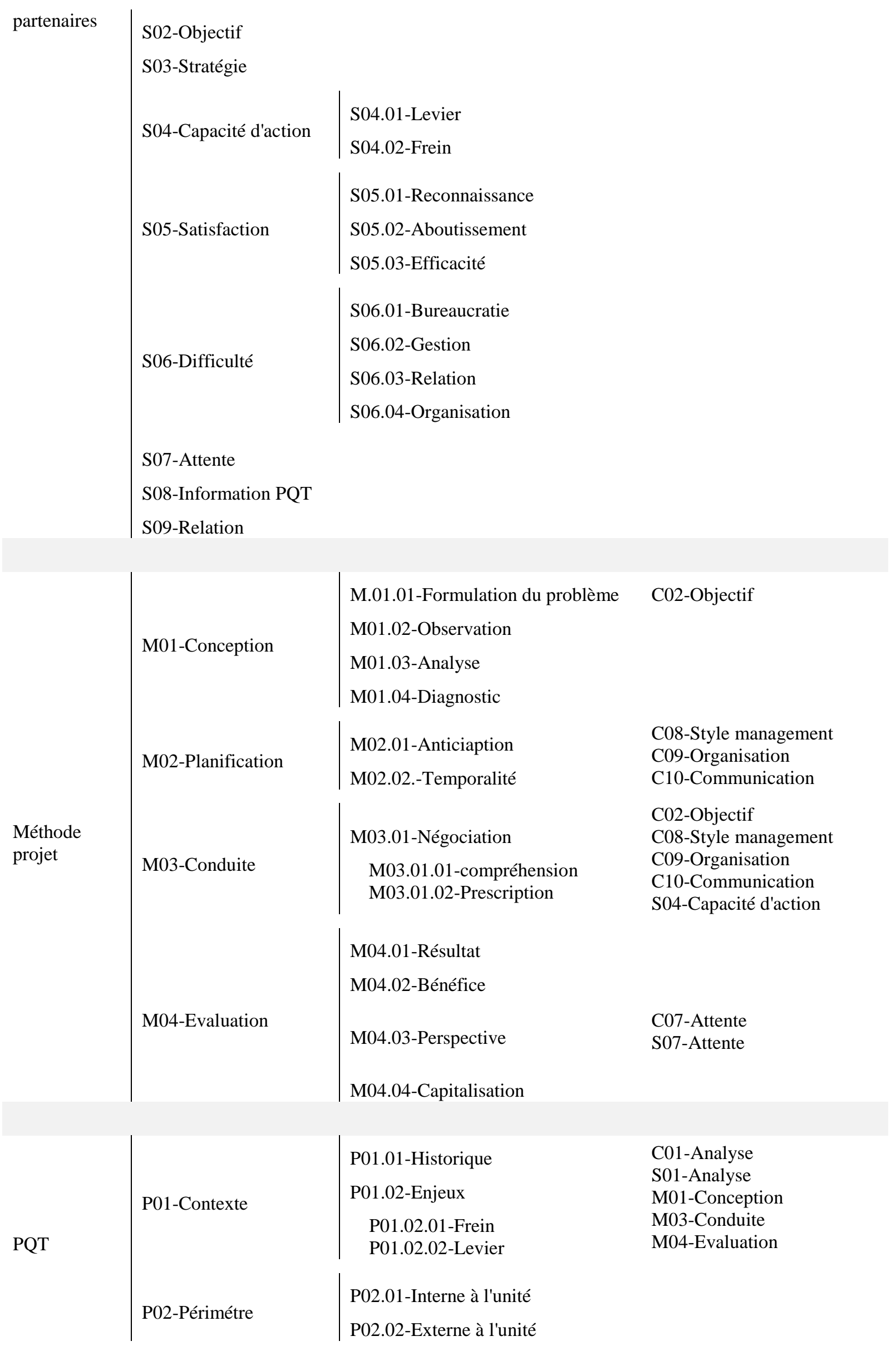




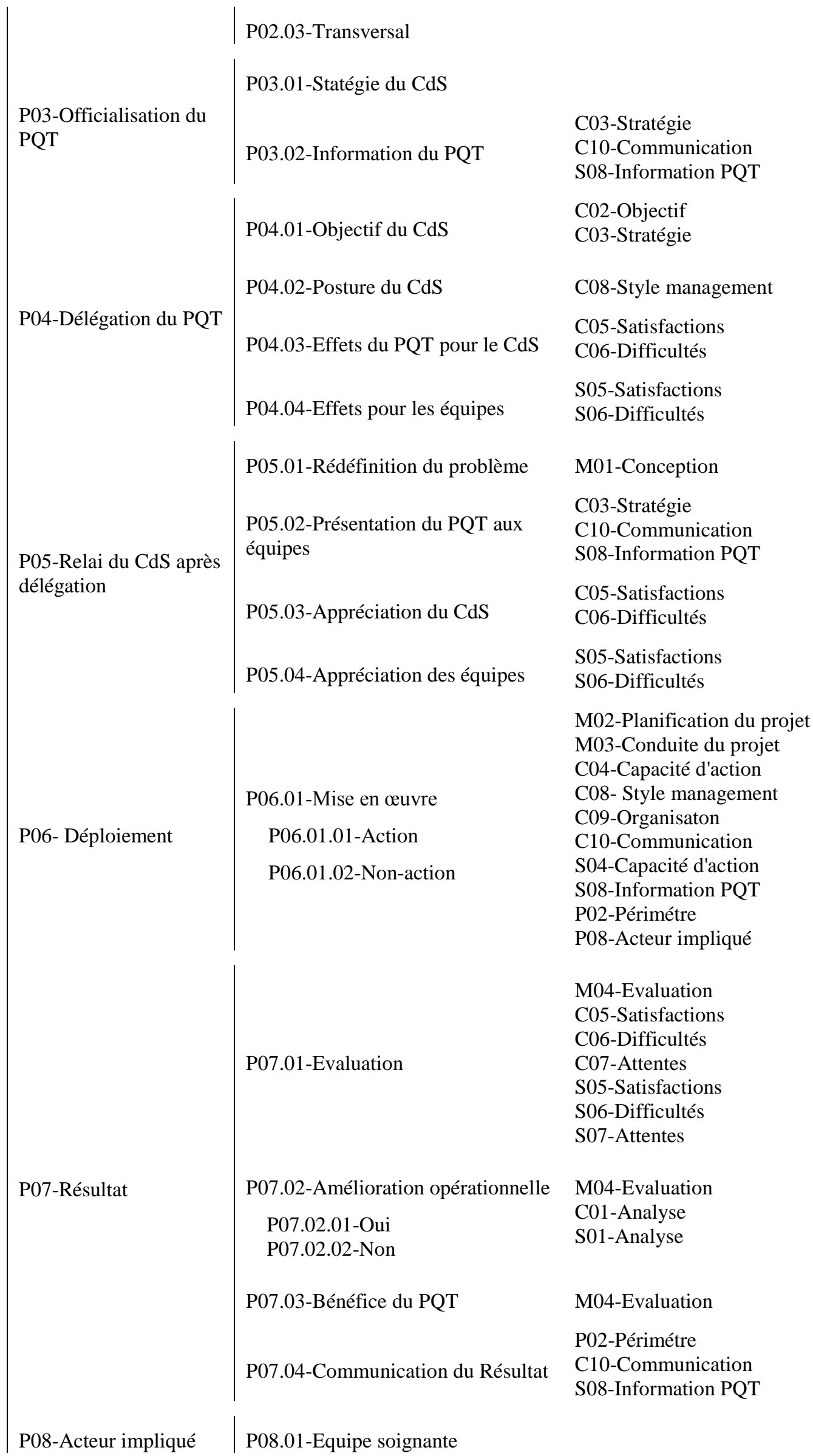


P08.02-Equipe médicale

P08.03-Hiérarchie soignante

P08.04-Syndicat

P08.05-Administratif/Direction

Tableau 2 : Intitulé des PQT et orientations relationnelles

\begin{tabular}{|c|c|c|c|c|}
\hline \multirow[b]{2}{*}{ PQT - Intitulé } & \multirow{2}{*}{$\begin{array}{l}\text { Métier } \\
\text { du } \\
\text { soin }\end{array}$} & \multicolumn{3}{|c|}{ Dimension } \\
\hline & & Institutionnelle & Transversale & Proximité \\
\hline $\begin{array}{l}01 \text { - Améliorer la confidentialité } \\
\text { à l'accueil des consultations }\end{array}$ & IDE & Attente & & Attente \\
\hline $\begin{array}{l}02 \text { - Hiérarchiser la réception des } \\
\text { examens de laboratoire à la } \\
\text { réception. }\end{array}$ & MT & & Intercompréhension & $\begin{array}{l}\text { Asymétrie } \\
\text { relationnelle }\end{array}$ \\
\hline $\begin{array}{l}03 \text { - Gérer les repas pour les } \\
\text { consultations longues }\end{array}$ & MT & Intercompréhension & & Prescription \\
\hline $\begin{array}{l}04 \text { - Réduire le retard des } \\
\text { consultations pour les patients } \\
\text { handicapés }\end{array}$ & IDE & & Intercompréhension & Intercompréhension \\
\hline $\begin{array}{l}05 \text { - Améliorer l'organisation du } \\
\text { travail et l'ambiance des équipes } \\
\text { pour une qualité de prise en } \\
\text { charge des patients }\end{array}$ & IDE & & & Intercompréhension \\
\hline $\begin{array}{l}06 \text { - Intégrer les transmissions } \\
\text { infirmières dans l'amplitude des } \\
12 \text { heures de travail }\end{array}$ & IDE & Attente & & Attente \\
\hline $\begin{array}{l}07 \text { - Améliorer le mode de } \\
\text { transmission professionnelle dans } \\
\text { le dossier du patient }\end{array}$ & Rééduc & & & Intercompréhension \\
\hline $\begin{array}{l}08 \text { - Améliorer la propreté des } \\
\text { locaux }\end{array}$ & IDE & & & Prescription \\
\hline $\begin{array}{l}09 \text { - Mettre en place la traçabilité } \\
\text { de l'entretien pour la certification }\end{array}$ & MT & Intercompréhension & & Prescription \\
\hline $\begin{array}{l}10 \text { - Améliorer la fluidité dans } \\
\text { l'utilisation des box de } \\
\text { consultation }\end{array}$ & IDE & Attente & & Prescription \\
\hline $\begin{array}{l}11 \text { - Améliorer les relations de } \\
\text { travail dans une équipe pluri- } \\
\text { professionnelle }\end{array}$ & IDE & & & $\begin{array}{l}\text { Asymétrie } \\
\text { relationnelle }\end{array}$ \\
\hline $\begin{array}{l}12 \text { - Fiabiliser la prescription } \\
\text { médicale et la traçabilité de l'acte }\end{array}$ & IDE & Attente & & Intercompréhension \\
\hline $\begin{array}{l}13 \text { - Optimiser la collaboration } \\
\text { interprofessionnelle }\end{array}$ & IDE & Intercompréhension & & Intercompréhension \\
\hline $\begin{array}{l}14 \text { - Améliorer les connaissances } \\
\text { des acteurs sur le processus de } \\
\text { stérilisation du matériel } \\
\text { chirurgical }\end{array}$ & MT & Attente & & Intercompréhension \\
\hline
\end{tabular}




\begin{tabular}{|c|c|c|c|c|}
\hline $\begin{array}{l}15-\text { Conserver l'expertise } \\
\text { professionnelle dans le projet de } \\
\text { mutualiser } 3 \text { équipes }\end{array}$ & IDE & Attente & & Attente \\
\hline $\begin{array}{l}16 \text { - Pérenniser une organisation } \\
\text { des astreintes sur le volontariat }\end{array}$ & Rééduc & Attente & Intercompréhension & Intercompréhension \\
\hline $\begin{array}{l}17 \text { - Favoriser la mobilité inter- } \\
\text { unité au sein du pôle }\end{array}$ & IDE & Attente & & Attente \\
\hline $\begin{array}{l}\text { Capacité de coordination } \\
\text { collective du CdS }\end{array}$ & & & & \\
\hline $\begin{array}{l}\text { Intercompréhension } \\
\text { organisationnelle }\end{array}$ & & 3 & 3 & 7 \\
\hline Asymétrie relationnelle & & & & 2 \\
\hline Prescription organisationnelle & & & & 4 \\
\hline Attente d'opportunité & & 8 & & 4 \\
\hline Total & & 11 & 3 & 17 \\
\hline
\end{tabular}

IDE : Infirmer ; MT : Médicotechnique ; Rééduc : Rééducateur

Figure 1 : Orientation du PQT dans trois dimensions relationnelles

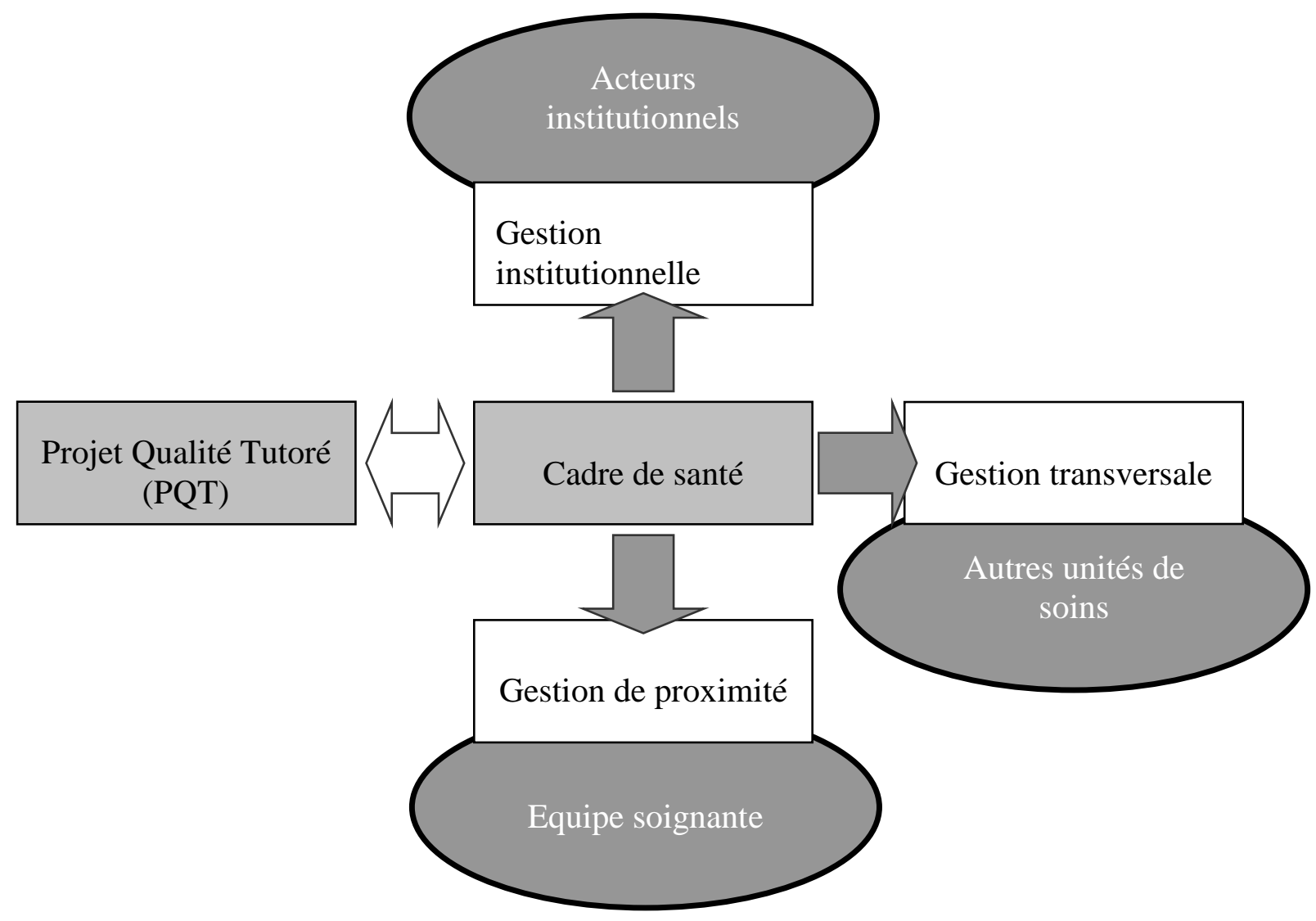

